\title{
Quantitative Assessment of Resting State for Mild Cognitive Impairment Detection: A Functional Near- Infrared Spectroscopy and Deep Learning Approach
}

\author{
Dalin Yang \\ Pusan National University \\ Keum-Shik Hong ( $\nabla$ kshong@pusan.ac.kr) \\ Pusan National University https://orcid.org/0000-0002-8528-4457
}

\section{Research}

Keywords: functional near-infrared spectroscopy, mild cognitive impairment, Alzheimer's disease, resting state, functional connectivity, convolutional neural network, transfer learning.

Posted Date: September 8th, 2020

DOI: https://doi.org/10.21203/rs.3.rs-69376/v1

License: (c) (1) This work is licensed under a Creative Commons Attribution 4.0 International License.

Read Full License

Version of Record: A version of this preprint was published at Journal of Alzheimer's Disease on March 23rd, 2021. See the published version at https://doi.org/10.3233/JAD-201163. 


\title{
Quantitative assessment of resting state for mild cognitive impairment detection: A functional near-infrared spectroscopy and deep learning approach
}

\author{
Dalin Yang ${ }^{1}$ and Keum-Shik Hong ${ }^{1, *}$ \\ Department of Cogno-Mechatronics Engineering, Pusan National University; 2 \\ Busandaehak-ro, Guemjeong-gu, Busan 46241, Republic of Korea \\ Emails: kshong@pusan.ac.kr \\ * Corresponding author:
}

\begin{abstract}
Background: Mild cognitive impairment (MCI) is considered a prodromal stage of Alzheimer's disease, which is the sixth leading cause of death in the United State. Early diagnosis of MCI can allow for treatment to improve cognitive function and reduce modifiable risk factors. Currently, the combination of machine learning and neuroimaging plays a role in identifying and understanding neuropathological diseases. However, some challenges still remain, and these limitations need to be optimized for clinical MCI diagnosis. Methods: In this study, for stable identification with functional near-infrared spectroscopy (fNIRS) using the minimum resting-state time, nine different measurement durations (i.e., 30, 60, 90, 120, 150, 180, 210, 240, and 270 s) were evaluated based on $30 \mathrm{~s}$ intervals using a traditional machine learning approach and graph theory analysis. The machine learning methods were trained using temporal features of the resting-state fNIRS signal and included linear discriminant analysis (LDA), support vector machine, and K-nearest neighbor (KNN) algorithms. To enhance the diagnostic accuracy, feature representation- and classification-based transfer learning (TL) methods were used to detect MCI from the healthy controls through the input of connectivity maps with 30 and $90 \mathrm{~s}$ durations. Results: As in the results of the traditional machine learning and graph theory analysis, there was no significant difference among the different time
\end{abstract}


windows. The accuracy of the conventional machine learning methods ranged from $55.76 \%(\mathrm{KNN}, 120 \mathrm{~s})$ to $67.00 \%(\mathrm{LDA}, 90 \mathrm{~s})$. The feature representation-based TL showed improved accuracy in both the 30 and 90 s cases (i.e., mean accuracy of $30 \mathrm{~s}$ : $79.37 \%$, mean accuracy of $30 \mathrm{~s}: 74.05 \%)$. Notably, the classification-based TL method achieved the highest accuracy of $97.01 \%$ using the VGG19 pre-trained CNN model trained with the $30 \mathrm{~s}$ duration connectivity map. Conclusion: The results indicate that a $30 \mathrm{~s}$ measurement of the resting state with fNIRS could be used to detect MCI. Moreover, the combination of neuroimaging (e.g., functional connectivity maps) and deep learning methods (e.g., CNN and TL) may be considered as novel biomarkers for clinical computer-assisted MCI diagnosis.

Keywords: functional near-infrared spectroscopy, mild cognitive impairment, Alzheimer's disease, resting state, functional connectivity, convolutional neural network, transfer learning. 


\section{Background}

Alzheimer's disease (AD) is the most common type of dementia, and it gradually but certainly influences the patient's memory and cognitive, mental, and language abilities [1]. In the late stage of $\mathrm{AD}$ (i.e., the severe stage), the symptoms have an increasing impact on the patient's motor and physical abilities, requiring around-the-clock care [2]. Typically, people with AD survive an average of four to eight years after diagnosis; however, some $\mathrm{AD}$ patients can live as long as 20 years. $\mathrm{AD}$ and related neurodegenerative diseases are arguably considered the most dreaded maladies of the aged [3]. None of the available pharmacologic or non-pharmacologic therapies are able to slow or stop the destruction of neurons caused by AD symptoms [2]. Mild cognitive impairment (MCI) is a transitional state between healthy aging and AD [4]. Researchers believe that treatment in the early stages (i.e., MCI) of the AD continuum may be effective for preventing the progression of $\mathrm{AD}$ and sustaining brain function [2]. Therefore, timely diagnosis of MCI from the healthy control (HC) presents an opportunity for interventions to improve cognitive function and reduce the modifiable risk factors implicated in AD progression [5].

Currently, the principal MCI diagnosis tools available to clinical doctors for making professional judgments rely on information obtained from the patient's medical history, mental status examination, imaging studies, and blood tests [6]. In comparison to subjective characteristics (i.e., the medical history and mental status examination), biomarker tests such as brain imaging have the potential to provide a quantitative clinical determination for suspected MCI. In particular, when assisted by computer science and mathematics, the use of brain imaging has provided the ability to understand the neural destruction caused by MCI in vivo [7]. 
There are two types of brain imaging techniques: (1) hemodynamic-metabolic types such as single-photon emission tomography (SPECT), positron emission tomography (PET), functional magnetic resonance imaging (fMRI), and functional near-infrared spectroscopy (fNIRS); and (2) electric-magnetic types, which include electroencephalography (EEG) and magnetoencephalography (MEG) [8]. PET and SPECT are based on the principle of radioactive isotopes. This characteristic has restricted their application in children and pregnant women [9]. Generally, brain imaging using electric-magnetic techniques provides high temporal resolution, but these methods are susceptible to motor artifacts and environmental interference [10]. Moreover, some of these techniques (e.g., EEG) also lack sufficient spatial resolution $(>1 \mathrm{~cm})$ [11]. In recent years, the use of fMRI has achieved valuable advancements in understanding neurological diseases [12]. However, the physically constrained participants must also be exposed to a loud noise environment [13]. Additionally, owing to the effects of the electric and magnetic fields, these brain imaging modalities cannot be applied simultaneously with brain stimulation (e.g., transcranial electrical stimulation) for further rehabilitation treatment [14]. In essence, fNIRS is an alternative to conventional hemodynamic response-based neuroimaging techniques that promises to shed additional light on functional brain activity in environments previously inaccessible to fMRI [15]. Similarly, fNIRS can measure concentration changes in oxygenated $(\Delta \mathrm{HbO})$ and deoxygenated $(\triangle \mathrm{HbR})$ hemoglobin by shooting near-infrared light (i.e., wavelengths of $650 \mathrm{~nm}$ to $1000 \mathrm{~nm}$ ) into the brain tissue to monitor the brain activity. In comparison with other non-invasive neuroimaging modalities (i.e., fMRI, EEG, and MEG), fNIRS has the advantages of safety, lower cost, portability, tolerance of motion artifacts, good temporal resolution, and moderate spatial resolution [16]. Additionally, the development of initial 
dip detection [17-19], bundled-optodes configurations [20-23], and adaptive algorithms [24-27] have offered further opportunities to improve the temporal and spatial resolution of fNIRS.

Owing to the promising advancement of fNIRS, several studies have investigated the competence of fNIRS for the detection of hemodynamic changes in AD and MCI. Through studies over the past decade, researchers have demonstrated the feasibility of using fNIRS to identify reduced cerebral oxygenation either in the resting state or the task period, such as during word retrieval, memory tasks, motor tasks, and visuospatial perception [28]. In the resting-state cases, altered connectivity [29-31] and fluctuations [32-36] of cerebral oxygenation have been reported in MCI and AD groups. This difference is associated with the dysregulation of information integration in the patient's brain. In the task period cases, oxygenation hypoactivation has been found in the frontotemporal area of the MCI group during verbal fluency tasks [37-39]. Additionally, a reduced hemodynamic response has been reported in the prefrontal cortex of the MCI group when performing memory tasks [40-44]. Similarly, examination results during motor $[45,46]$ and visuospatial $[47,48]$ tasks also showed a difference between the dementia and healthy groups. This reveals that executive dysfunction and abnormal visuospatial perception may be underlying in the patient's brain. These findings, as mentioned above, are consistent with fMRI results indicating a disrupted functional network and decreased hemodynamic response in MCI pathology. This empirical evidence also indicates that fNIRS-based indicators could play a role in the identification of MCI from the HC. Therefore, it is essential to develop and examine the practicability of fNIRS-based biomarkers for the diagnosis of MCI, as these could assist the clinical doctor in making confident decisions. 
In our initial studies $[49,50]$, we assessed the use of fNIRS-based biomarkers to detect MCI from the $\mathrm{HC}$ when participants were performing different mental tasks, such as the $N$-back task, Stroop task, and verbal fluency task. Seven digital biomarkers were extracted from the fluctuating time series of $\Delta \mathrm{HbO}$ and $\Delta \mathrm{HbR}$ : the mean, slope, peak value, kurtosis, and skewness. The highest accuracy was $76.67 \%$, classified by a linear discriminant analysis (LDA) based on the $N$-back task and Stroop task. The imaging biomarkers were evaluated using a convolutional neural network $(\mathrm{CNN})$, which included a $t$-map, mean map, slope map, kurtosis map, skewness map, HbO map, and connectivity map. The highest accuracy was $98.61 \%$ for the slope map case during the $N$-back task. However, after our initial investigations, some challenges and limitations still needed to be addressed: (1) small datasets may lead to overfitting and local minima during the training of the deep learning model, (2) some of the patients may be limited or dislike performing several mental tasks for the examination, and (3) the lengthy procedure of the cognitive task may cause fatigue problems for the patients.

Therefore, based on the limitations mentioned above, we further quantitatively investigated the possibility of using the features extracted from resting-state fNIRS data over a short period to detect MCI from the HC with a transfer learning method. Five minutes of resting-state data were acquired from 24 subjects. The digital biomarkers were extracted to examine the performance of the different resting-state periods. LDA, support vector machine (SVM), and k-nearest neighbor (KNN) algorithms were used to classify the digital biomarkers. The connectivity map was analyzed as the input to the transfer learning method. We hypothesized that: (1) the transfer learning method could fine tune the model trained with the small MCI dataset and obtain excellent performance, (2) the connectivity map of the resting state would be a useful biomarker for MCI detection, and 
(3) using a shorter time window of the resting state could achieve good classification accuracy. To the best of the authors' knowledge, this is the first quantitative fNIRS study to examine the resting state for MCI detection using deep learning methods.

\section{Materials and Methods}

\section{Participants and system protocol}

Twenty-four subjects (15 MCI patients: one male and 14 females; nine matched HC participants: two males and seven females) were recruited. All of the participants were employed by the Pusan National University Hospital (Busan, Rep. of Korea). The recruited subjects satisfied the following conditions: (1) right-handed, (2) able to communicate in Korean, (3) of a similar age, and (4) similar educational backgrounds. Before the fNIRS measurements, the mental health of the subjects was evaluated using the Korean mini-mental state examination (K-MMSE) [51], Seoul Neuropsychological Screening Battery [52], and magnetic resonance imaging (MRI). The Pusan National University Institutional Review Board approved the experimental protocol. Before the experiment, all subjects had the entire content of the experiment explained to them and provided informed consent. Figure 1 shows the system flowchart of the experimental process.

\section{[Figure 1]}

\section{fNIRS measurements}

A multi-channel NIRSIT continuous wave system (OBELAB Inc., Rep. of Korea) with 24 emitters and 32 detectors (Figure 2a) was employed to measure the fNIRS signals with a sampling rate of $8.138 \mathrm{~Hz}$. The wavelengths used for the detection of $\Delta \mathrm{HbO}$ and $\Delta \mathrm{HbR}$ were 780 and $850 \mathrm{~nm}$, respectively. The inter-optodes distance was set at $15 \mathrm{~mm}$. In total, 
48 channels were distributed equidistantly on the prefrontal cortex. The channel configuration is illustrated in Figure $2 b$, which was set to be consistent with the international 10-20 EEG system with reference electrode location FpZ. Each channel was defined as an emitter-detector pair with a distance of $30 \mathrm{~mm}$. The experiment was conducted in a confined room to avoid disturbance. The participants were seated on a comfortable chair and asked to rest without unnecessary movement. The duration of the fNIRS resting-state measurement was approximately 5 min for each participant.

\section{[Figure 2]}

\section{Resting-state preprocessing}

In this study, $\Delta \mathrm{HbO}$ and $\Delta \mathrm{HbR}$ throughout the whole experiment were obtained from the raw optical density using the modified Bear-Lambert Law [53, 54]. A fourth-order Butterworth low- and high-pass filter with a cut-off frequency of $0.0018-0.15 \mathrm{~Hz}$ was applied to remove cardiac noise $(\sim 1.1 \mathrm{~Hz})$, respiration $(0.25 \mathrm{~Hz})$, and other physiological noise [55-57]. In addition, a detrending algorithm was used to remove shifts in the baseline. However, the slowly varying U-shaped noise $(<0.1 \mathrm{~Hz})$ associated with deep breath or slow motion and other global unknown noise $(<0.1 \mathrm{~Hz})$ could not be removed by the bandpass filer or low-/high-pass filter [58]. Therefore, after passing the signal through the filter, we also manually checked and removed the noise channel with the obvious abnormal fluctuation by the experience. All of the preprocessing procedures were analyzed offline using MATLAB ${ }^{\mathrm{TM}}$ software (MathWorks, Natick, Massachusetts).

\section{Temporal features and functional connectivity}

To identify MCI and examine the minimum required duration of the resting-state data, we selected six temporal features to conduct the classification: the mean of $\Delta \mathrm{HbO}$, mean of $\Delta \mathrm{HbR}$, standard deviation of $\Delta \mathrm{HbO}$, standard deviation $\Delta \mathrm{HbR}$, variance of $\Delta \mathrm{HbO}$, and 
variance of $\Delta \mathrm{HbR}$. Each feature was calculated for nine different time intervals (i.e., 0 $30 \mathrm{~s}, 0-60 \mathrm{~s}, 0-90 \mathrm{~s}, 0-120 \mathrm{~s}, 0-150 \mathrm{~s}, 0-180 \mathrm{~s}, 0-210 \mathrm{~s}, 0-240 \mathrm{~s}$, and 0-270 s).

The functional connectivity of the brain signals reveals the interaction among different brain regions, which shows the temporal correlations between spatially distant neural activity [59]. Pearson's correlation coefficients (r) were used to analyze the correlation between the temporal signals of each channel. The connectivity matrix consists of the underlying interhemispheric relationships in the prefrontal cortex. The connectivity matrices used in this study were divided into two types: (1) calculations for graph theory analysis, and (2) imaging biomarkers for the transfer learning method. In the first case (i.e., used for the graph theory analysis), the input of the functional connectivity depended on the different time intervals (i.e., 0-30 s, 0-60 s, 0-90 s, 0-120 s, 0-150 s, 0-180 s, 0$210 \mathrm{~s}, 0-240 \mathrm{~s}$, and $0-270 \mathrm{~s})$. For the transfer learning case, the connectivity matrices were calculated with fixed $30 \mathrm{~s}$ durations (i.e., 0-30 s, 30-60 s, 60-90 s, 90-120 s, 120$150 \mathrm{~s}, 150-180 \mathrm{~s}, 180-210 \mathrm{~s}, 210-240 \mathrm{~s}$, and 240-270 s) and $90 \mathrm{~s}$ duration (i.e., 0-90 s, 90-180 s, and 180-270 s) for each subject.

\section{Graph theory}

Generally, graph theory has been employed to characterize the network communication ability. The nodes of the network refer to the channels, and the correlation coefficient is defined as the edge for each channel pair. Typically, the calculated network parameters (i.e., global efficiency, local efficiency, and small-worldness) have been used to describe changes in brain network architecture, disease development, and brain evolution [60-62]. To quantify the network characteristics and assess the minimum necessary measurement duration, the global efficiency, local efficiency, and small-worldness were analyzed with an increased sparsity threshold range (0.5-0.9) with an interval of 0.1 to evaluate the 
different periods in the resting-state functional connectivity. The threshold was applied to remove spurious connections, defined as when the edge is lower than the threshold. In essence, the global efficiency $\left(E_{g l o b}\right)$ measures the integration of the network, which indicates the ability to transfer information over the entire measured brain network $(G)$. The global efficiency is defined in Equation 1. The local efficiency $\left(E_{l o c}\right)$ of the network represents the communication ability in a local region. As given in Equation 2, it can be calculated based on the global efficiency. The small-worldness $(S w)$ is an ensemble of the measured networks that refers to the high-transferability network using the shortest path distance. Practically, it can be measured based on a comparison (shown in Equation 3) between the average cluster coefficient $(K)$ and path length $\left(L_{i}\right)$ for a random network.

$$
\begin{gathered}
E_{g l o b}(G)=\frac{1}{N(N-1)} \sum_{j \neq i \in G} \frac{1}{d(i, j)}, \\
E_{l o c}(G)=\frac{1}{N} \sum_{i \in G} E_{g l o b}\left(G_{i}\right) \\
S w_{i}=\frac{K L_{\text {rand }}}{L_{i} C_{\text {rand }}}
\end{gathered}
$$

where $N$ refers to the nodes of the network, and $d(i, j)$ represents the length of the shortest path between two random nodes, $i$ and $j$. The direct neighbor of the $i^{\text {th }}$ node produces the local subgraph network $\left(G_{i}\right)$. The path length and clustering efficiency of the random network are given by $L_{\text {rand }}$ and $C_{\text {rand }}$, respectively.

\section{Classification algorithm}

The vital objective of the classification algorithm is to describe and predict unknown terms through training with the existing dataset. LDA, SVM, and KNN are the most widely used machine learning methods for brain disease identification [63]. The principle of LDA is the generation of hyper-planes to discriminate data from different classes. Similarly, the main function of the SVM is to construct a margin (i.e., support vectors) 
with a maximum boundary to generate a linear extrication hyper-plane. In contrast, KNN is a type of instance-based learning, in which the vote of its neighbors categorizes an object. In this study, we selected six temporal features (mean of $\Delta \mathrm{HbO}$, mean of $\Delta \mathrm{HbR}$, standard deviation of $\Delta \mathrm{HbO}$, standard deviation of $\Delta \mathrm{HbR}$, variance of $\Delta \mathrm{HbO}$, and variance of $\Delta \mathrm{HbR})$ of the fNIRS signals with different durations $(0-30 \mathrm{~s}, 0-60 \mathrm{~s}, 0-90 \mathrm{~s}$, 0-120 s, 0-150 s, 0-180 s, 0-210 s, 0-240 s, and 0-270 s) to classify MCI from the HC. Five runs of a 5-fold cross-validation were applied for each classifier (i.e., LDA, SVM, and $\mathrm{KNN}$ ).

\section{[Figure 3]}

With the development of deep learning and neural imaging techniques, computer-assisted clinical diagnosis methods have achieved massive progress. In particular, CNNs have realized huge successes in brain disease identification. However, many challenges remain, such as the cost of neural imaging examinations, limited datasets for rare diseases, and long procedures for clinical diagnosis [64]. It is difficult to accumulate a sufficient dataset to train CNN models. To avoid overfitting and the lower generalization power of a small dataset, transfer learning is a good solution [65]. In concept, transfer learning is used as a pre-trained model for fine tuning the new model to extract features or classify unknown objects [66], as shown in Figure 3. In this study, we employed two different transfer learning strategies: feature representation-based transfer learning (FRTL) and classification-based transfer learning (CTL). In the FRTL case (i.e., Figure 3a), the pretrained CNN model was used to extract the features from the connectivity map, and the extracted features were used as the input to the SVM to classify MCI from the HC. Normally, it is suggested to choose deeper convolutional layers to extract features, as deeper layers always contain more detailed characteristics. In contrast, CTL (Figure 3b) 
applies the pre-trained model to fine tune the new model by altering the parameters of the input and output layers directly. In this study, seven pre-trained CNN models (i.e., VGG 16, VGG19, Alexnet, Resnet18, Resnet50, Resnet101, and Densenet 201) were used for conducting the transfer learning. To evaluate the performance, confusion metrics were calculated after the classification, which include true positive (TP), false negative (FN), true negative $(\mathrm{TN})$, and false positive (FP) occurrence. TP refers to the number of MCI cases classified correctly. FN represents the number of HC misclassified. Similarly, the number of $\mathrm{HC}$ detected correctly is the TN, while FP is the number of MCI cases that were misclassified. Based on the confusion metrics, the accuracy, recall, precision, and F1-score could be calculated as follows:

$$
\begin{gathered}
\text { Accuracy }=\frac{\mathrm{TP}+\mathrm{TN}}{\mathrm{TP}+\mathrm{TN}+\mathrm{FP}+\mathrm{FN}}, \\
\text { Recall }=\frac{\mathrm{TP}}{\mathrm{TP}+\mathrm{FN}}, \\
\text { Precision }=\frac{\mathrm{TP}}{\mathrm{TP}+\mathrm{FP}}, \\
\mathrm{F} 1 \text { - score }=2 \times \frac{\text { Precision } \times \text { Recall }}{\text { Precision+Recall }} .
\end{gathered}
$$

\section{Results}

\section{Demographics and clinical score}

Table 1 summarizes the demographic and clinical characteristics of all participants. The statistical difference (i.e., $p$-value), mean, and standard deviation were calculated for the parameters of gender, education, age, and K-MMSE score. The statistical analysis was conducted using two independent sample $t$-tests with a significance level of 0.05 . In the age case, the $p$-value was equal to 0.36 , which indicates a non-significant difference between the MCI and HC groups. Therefore, the participants in the two groups are age matched. Similarly, the MCI and HC had matching educational backgrounds ( $p$-value $=$ 
$0.36>0.05$ ). The averaged K-MMSE score shows that the MCI group (i.e., 25.13) had a lower score than the HC group (i.e., 27.22), which indicates the decreased cognitive state in the MCI group. To interpret the K-MMSE values, normal cognition is categorized as a score of 24 or greater (with a maximum of 30). The mean of the K-MMSE scores in the MCI group is higher than 24. Interestingly, the statistical analysis results also revealed a non-significant difference $(p$-value $=0.49>0.05)$ between the two groups.

\section{[Table 1]}

\section{Temporal feature classification results}

Rather than using a statistical analysis to identify the differences between the two groups, individual identification using the machine learning method may offer promising advantages for clinical diagnosis. Figure 4 shows the classification accuracy obtained with three different machine learning algorithms (i.e., LDA, SVM, and KNN) for the nine measurement durations. The mean accuracies of the LDA, SVM, and KNN algorithms are $63.27 \%, 58.93 \%$, and $57.71 \%$, respectively. Moreover, there is no significant improvement in accuracy with increasing measurement duration in any of the classification cases. This suggests that using the $30 \mathrm{~s}$ resting-state measurement (LDA: 62.56\%, SVM: 58.82\%, and KNN: 57.54\%) may achieve a similar classification performance as using the 270 s measurement (LDA: 62.96\%, SVM: $57.14 \%$, and KNN: $57.14 \%)$. Interestingly, the highest classification accuracy among the nine measurement durations appears at the $90 \mathrm{sec}$ in the LDA (i.e., 67.00\%) and KNN (i.e., 60.20\%) classification accuracy.

[Figure 4] 


\section{Functional connectivity map}

The connectivity maps are generated by the functional connectivity matrix, which demonstrates the temporal relationship among the 48 channels. Figure 5 illustrated the functional connectivity of the MCI and $\mathrm{HC}$ from $30 \mathrm{sec}$ to $270 \mathrm{sec}$ for the $\Delta \mathrm{HbO}$ and $\Delta \mathrm{HbR}$ cases. After the time window extends longer than $90 \mathrm{~s}$, the functional connectivity maps for all four categories (i.e., $\Delta \mathrm{HbO}$ of $\mathrm{MCI}, \Delta \mathrm{HbO}$ of $\mathrm{HC}, \Delta \mathrm{HbR}$ of $\mathrm{MCI}$, and $\Delta \mathrm{HbR}$ of HC) exhibit similar patterns. However, The HC group displays a stronger functional connectivity than the MCI group, which can be observed for all the measurement durations.

\section{[Figure 5]}

\section{Graph theory analysis}

In this study, graph theory was employed to further evaluate the functional connectivity network and quantify the difference between different measurement durations. Figure 6 shows the global network efficiency, local network efficiency, and small-worldness for nine measurement durations and four (i.e., $\Delta \mathrm{HbO}$ of MCI, $\triangle \mathrm{HbO}$ of $\mathrm{HC}, \Delta \mathrm{HbR}$ of MCI, and $\triangle \mathrm{HbR}$ of $\mathrm{HC}$ ). The results for the global (Figure 6a) and local (Figure 6b) efficiencies do not exhibit a significant difference with increasing measurement duration. In Figure $6 \mathrm{c}$, the $30 \mathrm{~s}$ duration has a lower small-worldness than the $90 \mathrm{~s}$ duration. However, after $90 \mathrm{~s}$, the small-world results remain consistent. This result is based on the functional connectivity map and the temporal feature classification result. It indicates that the resting-state duration required for stable analysis of the difference between the MCI and HC groups can be set as $90 \mathrm{~s}$. However, based on the results of the temporal classification 
accuracy and functional connectivity, a measurement duration of $30 \mathrm{~s}$ is also able to discriminate the difference between the MCI and $\mathrm{HC}$ groups.

\section{[Figure 6]}

\section{Feature representation-based transfer learning}

To examine the minimum measurement duration, we further selected $30 \mathrm{~s}$ and $90 \mathrm{~s}$ as time windows for the generation of the connectivity map. The total resting state period $(270 \mathrm{~s})$ was thus divided into nine sections $(270 \div 30=9)$ and three sections $(270 \div 90=$ 3), respectively. Therefore, the inputs to the transfer learning were 216 (i.e., $9 \times 24=216$ ) and 72 (i.e., $3 \times 24=72$ ), respectively. The input size of the first layer was normalized according to the requirement of each $\mathrm{CNN}$ model. The dataset was divided to designate 80 and $70 \%$ as the training datasets in two cases. Tables 2 and 3 present the classification results of the $\Delta \mathrm{HbO}$ and $\Delta \mathrm{HbR}$ biomarkers, respectively, with seven pre-trained $\mathrm{CNN}$ models for the input map generated using $30 \mathrm{~s}$ periods. The accuracy obtained using $\Delta \mathrm{HbO}$ ranged from $73.58 \%$ (with Resnet 101 ) to $83.82 \%$ (with Resnet 50 ); the average accuracy was $80.36 \%$. As presented in Table 3, the mean accuracy of the classification based on $\Delta \mathrm{HbR}$ (i.e., $78.38 \%$ ) was lower than that based on $\Delta \mathrm{HbO}$, and it ranged from $69.65 \%$ (with Alexnet) to $85.92 \%$ (with Densenet 201). For the $90 \mathrm{~s}$ time window, the accuracies of the classifications using both $\Delta \mathrm{HbO}$ (i.e., 74.36\%) and $\Delta \mathrm{HbR}$ (i.e., $73.75 \%$ ) were lower than those for the $30 \mathrm{~s}$ time window, as listed in Tables 4 and 5 .

\section{[Table 2]}

[Table 3]

[Table 4]

[Table 5] 


\section{Classification-based transfer learning}

Owing to the long training time cost and the similar classification performance obtained with the FRTL, in this section, only the three pre-trained CNN models with the fewest layers (i.e., VGG 16, VGG 19, and Alexnet) were considered. The classification results of these three models with $\Delta \mathrm{HbO}$ and $\Delta \mathrm{HbR}$ for the $30 \mathrm{~s}$ and $90 \mathrm{~s}$ measurement durations are summarized in Tables 6-9. In comparison to the performance of the FRTL, the accuracy of the CTL is improved, especially for the $30 \mathrm{~s}$ time window (i.e., $\Delta \mathrm{HbO}$ : 88.74\%, $\triangle \mathrm{HbR}: 93.17 \%$ ). In particular, the highest accuracy reaches $97.01 \%$ (with VGG 19). The accuracy is slightly increased with the $90 \mathrm{~s}$ time window (i.e., $\Delta \mathrm{HbO}: 83.74 \%$, $\Delta$ HbR: $77.31 \%$ ). Furthermore, the $80 \%$ training set always presents a higher accuracy than the $70 \%$ training set in the 30 s classification. Interestingly, this trend does not occur when the $90 \mathrm{~s}$ time window is used.

\section{[Table 6]}

\section{[Table 7]}

\section{[Table 8]}

\section{[Table 9]}

\section{Discussion}

In this study, our goal was to evaluate the minimum resting-state fNIRS signal and investigate the possibility of using transfer learning to detect $\mathrm{MCI}$ from the $\mathrm{HC}$ based on the resting-state fNIRS. To the best of the authors' knowledge, this is the first study to examine the minimum resting-state duration required for MCI detection using fNIRS signals. In addition, we believe it is the first study to apply transfer learning for individual classification of MCI based on the resting-state fNIRS signal. The cost of the neural 
imaging technique, difficulty of the signal acquisition for patients, and limited data available for rare diseases have always been major challenges for the application of computer-assisted brain disease diagnosis. Our system provides a solution for the comfortable and accurate detection of individual MCI from the HC, which is obtained using the transfer learning classification method with an input of the minimum restingstate fNIRS data.

MCI and dementia are multifaceted diseases, which cause accumulated pathological brain injury leading to decline in progressive motor, cognitive, and language abilities. The wellestablished biomarkers are neurodegeneration, amyloid plaques, and neurofibrillary tangles [67]. With the development of non-invasive neuroimaging techniques, the use of imaging biomarkers has emerged owing to their promising advantages of ease of signal measurement, safety, non-invasive nature, and low cost. In comparison to fMRI, EEG, PET, and SPECT, fNIRS as a novel non-invasive neural imaging technique is more environmentally unconstrained and has moderate temporal and spatial resolution. Thus, it is a promising tool for assessing biomarkers for brain disease diagnosis.

Empirical fNIRS studies have investigated the differences between MCI and the HC [28]. Some of the literature $[41,44]$ has stated that less hemodynamic response appears in the frontal and parietal regions of the MCI and $\mathrm{AD}$ groups in comparison to the $\mathrm{HC}$ group when performing working memory tasks. Similarly, in the resting state [30,61], and during other cognitive tasks [38], the hypoactivation measured by fNIRS has also been reported in statistical analyses at the group level. As such, the reduced hemodynamic response and functional connectivity were also obtained in our study, and the MCI group exhibited lower functional connectivity than the HC group (Figure 5). Similarly, this finding is consistent with the literature on fMRI [67] and EEG [68]. These experiential 
studies provide interpretability of the dysregulation and neurodegeneration of MCI, and also verify the feasibility of using fNIRS as a biomarker for MCI detection.

Mass-univariate analysis (i.e., statistical parametric mapping) plays a vital role in the determination of abnormal hemodynamic responses and the neurofunctional difference between the patient and control groups [69]. However, for individual MCI diagnosis, the statistical analysis cannot be easily applied [70]. Similarly, in our initial evaluation study [50], the statistical analysis outcomes showed inconsistencies with the results of the individual classifications. Therefore, it is essential to validate the biomarker results based on individual classifications before the established biomarker can be applied as a clinical assistance tool.

In our previous study [49,50], digital biomarkers (i.e., mean, slope, peak, kurtosis, and skewness) and imaging biomarkers (i.e., mean map, slope map, HbO map, skewness map, kurtosis map, $t$-map, and connectivity map) during mental tasks (i.e., the $N$-back task, Stroop task, and VFT) were evaluated for MCI detection using LDA and CNN. The highest accuracies for the digital and imaging biomarkers were $76.67 \%$ (with LDA) and 98.61\% (with CNN), respectively. The biomarkers in our initial work are comparable to the established biomarkers, such as PET, SPECT, and cerebrospinal fluid [71]. In addition, a novel study [31] reported that using a K-mean clustering analysis of the functional connectivity index could detect MCI with a sensitivity of $84 \%$ and specificity of $70 \%$. In comparison to those studies, the current study used a resting-state signal with the minimum time window, in which the most salient feature is the convenience of data acquisition for MCI patients without performing any tasks. In addition, the transfer learning method offers the opportunity for using small datasets, which may occur owing 
to the limitation of cost or patient numbers, to make decisions based on deep learning methods.

In fNIRS, which is a novel and promising technique, the necessary resting-state acquisition duration for accurate and stable MCI detection remains largely unknown. In the fMRI case, the minimum scanning time should be longer than 5 min to achieve stable functional connectivity. One study on a child via the fNIRS resting state [72] demonstrated that 2.5 min may be the minimum time for accurate measurement. In our study, this is consistent with the results of the functional connectivity observations (i.e., Figure 5), in which the measurement durations of longer than 2.5 min show stable patterns. However, interestingly, the quantitative assessments based on the classification (i.e., LDA, SVM, and KNN) and graph theory analysis (i.e., global efficiency, local efficiency, and small-worldness) show that measurement durations of at least 1.5 min have similar performance (i.e., Figures 4 and 6). In particular, the classification results demonstrate that a measurement duration of 0.5 min could also achieve satisfactory results. This is why $30 \mathrm{~s}$ and $90 \mathrm{~s}$ time windows were selected to divide the resting-state data for the transfer learning classification.

To avoid overfitting and local minima during the classification due to the small dataset, transfer learning was used to solve this problem for the traditional deep learning. In the FRTL case, we employed seven pre-trained CNN models to extract the features. Each pretrained model have trained by millions of images and had the ability to classify the 1000 categories. However, each well-established CNN models have different CNN structures (layers). Nevertheless, the performance of the FRTL with seven pre-trained CNN models exhibited no significant difference in this study. In addition, the performance of the $80 \%$ training set was generally higher than that of the $70 \%$ training 
set. One possible reason is that some of the datasets were not sufficient to fine tune the pre-trained $\mathrm{CNN}$ model. However, the $90 \mathrm{~s}$ time window could obtain a more stable performance than the $30 \mathrm{~s}$ window during the analysis using traditional machine learning methods and the graph theory analysis. Both transfer learning results classified using the $90 \mathrm{~s}$ duration datasets showed a lower accuracy than those classified using the $30 \mathrm{~s}$ duration datasets. This may be due to the imbalance in the input data.

\section{Limitation}

Although the CTL provided satisfactory diagnosis accuracy (maximum accuracy: 97.01\%), there are some limitations to this technique, and future directions must be discussed. First, owing to the insufficient resting-state data, the clipped sections with durations of $30 \mathrm{~s}$ and $90 \mathrm{~s}$ were unbalanced. Increasing and balancing the input numbers may further improve the classification accuracy. Second, owing to the long time required for the CTL procedure and the similar performances obtained with the FRTL, we only selected three pre-trained CNN models to differentiate the MCI from the $\mathrm{HC}$ group using CTL. Therefore, a future study could examine the rest of the models with the assistance of a supercomputer to check whether the higher performance may achieve or not. Finally, the prefrontal cortex has the benefit of no overlying hair, which can reduce the scattering and attenuation effects. This study only examines the resting state in the prefrontal cortex. However, the default mode network may have a high possibility of identifying biomarkers useful for MCI detection.

\section{Conclusion}

In this study, we assessed the minimum resting-state duration required to obtain an accurate functional connectivity map. Moreover, a novel method to identify MCI from 
the HC using transfer learning methods was proposed. The traditional machine learning and graph theory analysis results for nine measurement intervals (i.e., 0-30 s, 0-60 s, 0$90 \mathrm{~s}, 0-120 \mathrm{~s}, 0-150 \mathrm{~s}, 0-180 \mathrm{~s}, 0-210 \mathrm{~s}, 0-240 \mathrm{~s}$, and 0-270 s) illustrated that there was no significant difference in these measurement durations. The transfer learning results demonstrated that the minimum time window of $30 \mathrm{~s}$ in the resting state could achieve good accuracy (maximum accuracy: 97.01\%) for MCI detection. This investigation can provide a reference for future studies to select the minimum measurement duration of the resting state for fNIRS. In addition, these methodologies could present a novel approach for MCI detection, especially for cases with small datasets. Moreover, the proposed biomarkers of the resting state connectivity map with the combined deep learning method could be used as the tool to assist the clinical MCI diagnosis.

\section{Abbreviations}

AD: Alzheimer's disease; MCI: mild cognitive impairment; HC: healthy control; SPECT: single-photon emission tomography; PET: positron emission tomography; fMRI: functional magnetic resonance imaging; fNIRS: functional near infrared spectroscopy; ; EEG: electroencephalography; MEG: magnetoencephalography; HbO: oxy-hemoglobin; HbR: deoxy-hemoglobin; LDA: linear discriminant analysis; CNN: convolutional neural network; SVM: support vector machine; KNN: k-nearest neighbor (KNN);K-MMSE: Korean mini-mental state examination; MRI: magnetic resonance imaging; FRTL: feature representation-based transfer learning; CTL: classification-based transfer learning; TP: true positive, FN: false negative; TN: true negative; FP: false positive. 


\section{Author's Contributions}

Study design: DY; data acquisition: DY; analysis and interpretation: DY; manuscript drafting; KSH has conceived the idea, investigated the theoretical aspects of the work, supervised all the processes from the beginning, and finalized the manuscript. All authors have read and approved the final manuscript.

\section{Funding}

This work was supported by the National Research Foundation (NRF) of Korea under the auspices of the Ministry of Science and ICT, Korea (grant no. NRF2020R1A2B5B03096000).

\section{Acknowledgement}

We would like to special thank all participants for taking part in this study and the doctors of Pusan National University Hospital for helping us with recruitment of participants.

\section{Competing interests}

The authors declare that they have no competing interests.

\section{Consent for publication}

Not applicable

\section{Ethics approval and consent to participate}

This study was reviewed and approved by the Ethics Committee of the Pusan National University Hospital (H-1809-0190971) and all procedures involved were conducted in 
accordance with the latest version of the Declaration of Helsinki. Prior to experiments, written informed consent after receiving detailed information about the experiment procedure was obtained from the subjects.

\section{Data availability statement}

The datasets generated for this study are available from the corresponding author on reasonable request.

\section{Author's information}

Department of Cogno-Mechatronics Engineering, Pusan National University; 2 Busandaehak-ro, Guemjeong-gu, Busan 46241, Republic of Korea

Table 1. Demographic information and clinical characteristics of the participants

\begin{tabular}{llll}
\hline Characteristics & MCI $(\mathbf{n}=\mathbf{1 5})$ & HC $(\mathbf{n}=\mathbf{9})$ & $\boldsymbol{p}$-value \\
\hline Gender (Male/Female) & $1 / 14$ & $2 / 7$ & 0.44 \\
Education [years] (mean/std) & $11.2( \pm 4.81)$ & $10.56( \pm 2.88)$ & 0.36 \\
Age [years] (mean/std) & $69.27( \pm 7.09)$ & $68.33( \pm 4.69)$ & 0.36 \\
K-MMSE Score (mean/std) & $25.13( \pm 2.33)$ & $27.22( \pm 1.98)$ & 0.49 \\
\hline \hline
\end{tabular}

K-MMSE: Korea Mini-Mental State Examination: std: standard deviation; n: number of participants.

Table 2. Feature representation-based transfer learning classification results of different pre-trained $\mathrm{CNN}$ models using $30 \mathrm{~s}$ measurement duration for the resting state of $\Delta \mathrm{HbO}$

\begin{tabular}{|c|c|c|c|c|c|c|}
\hline \multirow{2}{*}{ CNN model } & \multirow{2}{*}{ Feature layer } & \multirow{2}{*}{ Dataset } & \multicolumn{4}{|c|}{ Averaged Performance(HbO)_30 sec } \\
\hline & & & Accuracy & Recall & Precision & F1-score \\
\hline \multirow{4}{*}{ VGG16 } & \multirow{2}{*}{ fc6 } & $80 \%$ training & $82.43 \%$ & $90.85 \%$ & $73.75 \%$ & $80.36 \%$ \\
\hline & & $70 \%$ training & $80.25 \%$ & $86.97 \%$ & $72.50 \%$ & $77.89 \%$ \\
\hline & \multirow{2}{*}{ fc7 } & $80 \%$ training & $81.62 \%$ & $82.03 \%$ & $82.50 \%$ & $81.85 \%$ \\
\hline & & $70 \%$ training & $82.92 \%$ & $81.66 \%$ & $85.83 \%$ & $83.37 \%$ \\
\hline \multirow{2}{*}{ Resnet50 } & \multirow{2}{*}{ fc1000 } & $80 \%$ training & $83.82 \%$ & $89.91 \%$ & $78.75 \%$ & $81.93 \%$ \\
\hline & & $70 \%$ training & $79.83 \%$ & $89.45 \%$ & $71.67 \%$ & $73.34 \%$ \\
\hline \multirow{2}{*}{ Resnet18 } & \multirow{2}{*}{ fc1000 } & $80 \%$ training & $84.82 \%$ & $88.51 \%$ & $80.00 \%$ & $83.29 \%$ \\
\hline & & $70 \%$ training & $83.00 \%$ & $87.89 \%$ & $77.50 \%$ & $81.16 \%$ \\
\hline
\end{tabular}




\begin{tabular}{|c|c|c|c|c|c|c|}
\hline \multirow{2}{*}{ Resnet101 } & \multirow{2}{*}{ fc 1000} & $80 \%$ training & $76.50 \%$ & $69.87 \%$ & $93.75 \%$ & $79.98 \%$ \\
\hline & & $70 \%$ training & $73.58 \%$ & $80.59 \%$ & $69.17 \%$ & $71.00 \%$ \\
\hline \multirow{2}{*}{ Densenet201 } & \multirow{2}{*}{ fc1000 } & $80 \%$ training & $76.04 \%$ & $76.78 \%$ & $78.75 \%$ & $76.26 \%$ \\
\hline & & $70 \%$ training & $82.25 \%$ & $86.75 \%$ & $77.50 \%$ & $80.19 \%$ \\
\hline \multirow{4}{*}{ VGG19 } & \multirow{2}{*}{ fc6 } & $80 \%$ training & $78.08 \%$ & $85.48 \%$ & $68.75 \%$ & $75.31 \%$ \\
\hline & & $70 \%$ training & $83.17 \%$ & $85.11 \%$ & $80.83 \%$ & $82.45 \%$ \\
\hline & \multirow{2}{*}{$\mathrm{fc} 7$} & $80 \%$ training & $79.91 \%$ & $79.82 \%$ & $85.00 \%$ & $81.11 \%$ \\
\hline & & $70 \%$ training & $79.25 \%$ & $86.51 \%$ & $72.50 \%$ & $75.55 \%$ \\
\hline \multirow{4}{*}{ Alexnet } & \multirow{2}{*}{ fc6 } & $80 \%$ training & $80.32 \%$ & $87.82 \%$ & $72.50 \%$ & $77.79 \%$ \\
\hline & & $70 \%$ training & $77.75 \%$ & $82.69 \%$ & $72.50 \%$ & $73.79 \%$ \\
\hline & \multirow{2}{*}{ fc7 } & $80 \%$ training & $79.33 \%$ & $87.09 \%$ & $71.25 \%$ & $76.21 \%$ \\
\hline & & $70 \%$ training & $82.33 \%$ & $81.28 \%$ & $86.67 \%$ & $83.19 \%$ \\
\hline \multicolumn{3}{|l|}{ Mean } & $80.36 \%$ & $84.35 \%$ & $77.58 \%$ & $78.80 \%$ \\
\hline
\end{tabular}

Table 3. Feature representation-based transfer learning classification results of different pre-trained CNN models using $30 \mathrm{~s}$ measurement duration for the resting state of $\Delta \mathrm{HbR}$

\begin{tabular}{|c|c|c|c|c|c|c|}
\hline \multirow{2}{*}{ CNN model } & \multirow{2}{*}{ Feature layer } & \multirow{2}{*}{ Dataset } & \multicolumn{4}{|c|}{ Averaged Performance(HbR)_30 sec } \\
\hline & & & Accuracy & Recall & Precision & F1-score \\
\hline \multirow{4}{*}{ VGG16 } & \multirow{2}{*}{ fc6 } & $80 \%$ training & $79.75 \%$ & $83.46 \%$ & $78.75 \%$ & $79.36 \%$ \\
\hline & & $70 \%$ training & $82.33 \%$ & $82.14 \%$ & $86.67 \%$ & $82.31 \%$ \\
\hline & \multirow{2}{*}{ fc7 } & $80 \%$ training & $84.07 \%$ & $89.69 \%$ & $80.00 \%$ & $77.25 \%$ \\
\hline & & $70 \%$ training & $74.58 \%$ & $89.60 \%$ & $59.17 \%$ & $67.34 \%$ \\
\hline \multirow{2}{*}{ Resnet50 } & \multirow{2}{*}{ fc1000 } & $80 \%$ training & $73.98 \%$ & $86.23 \%$ & $65.00 \%$ & $64.93 \%$ \\
\hline & & $70 \%$ training & $75.83 \%$ & $80.73 \%$ & $71.67 \%$ & $73.72 \%$ \\
\hline \multirow{2}{*}{ Resnet18 } & \multirow{2}{*}{ fc1000 } & $80 \%$ training & $85.32 \%$ & $89.03 \%$ & $82.50 \%$ & $83.46 \%$ \\
\hline & & $70 \%$ training & $85.92 \%$ & $90.48 \%$ & $80.83 \%$ & $84.79 \%$ \\
\hline \multirow{2}{*}{ Resnet101 } & \multirow{2}{*}{ fc1000 } & $80 \%$ training & $77.59 \%$ & $74.29 \%$ & $90.00 \%$ & $80.47 \%$ \\
\hline & & $70 \%$ training & $79.00 \%$ & $86.31 \%$ & $72.50 \%$ & $76.39 \%$ \\
\hline \multirow{2}{*}{ Densenet201 } & \multirow{2}{*}{ fc1000 } & $80 \%$ training & $83.03 \%$ & $92.48 \%$ & $71.25 \%$ & $80.37 \%$ \\
\hline & & $70 \%$ training & $82.92 \%$ & $89.98 \%$ & $75.83 \%$ & $80.19 \%$ \\
\hline \multirow{4}{*}{ VGG19 } & \multirow{2}{*}{ fc6 } & $80 \%$ training & $76.67 \%$ & $77.72 \%$ & $80.00 \%$ & $81.86 \%$ \\
\hline & & $70 \%$ training & $81.42 \%$ & $79.28 \%$ & $85.83 \%$ & $81.95 \%$ \\
\hline & \multirow{2}{*}{ fc7 } & $80 \%$ training & $76.00 \%$ & $83.80 \%$ & $71.25 \%$ & $77.16 \%$ \\
\hline & & $70 \%$ training & $73.17 \%$ & $85.93 \%$ & $58.33 \%$ & $64.80 \%$ \\
\hline \multirow{4}{*}{ Alexnet } & \multirow{2}{*}{ fc6 } & $80 \%$ training & $74.03 \%$ & $76.19 \%$ & $72.50 \%$ & $73.02 \%$ \\
\hline & & $70 \%$ training & $77.17 \%$ & $82.09 \%$ & $75.83 \%$ & $74.20 \%$ \\
\hline & \multirow{2}{*}{ fc7 } & $80 \%$ training & $69.65 \%$ & $93.57 \%$ & $43.75 \%$ & $50.60 \%$ \\
\hline & & $70 \%$ training & $75.08 \%$ & $79.38 \%$ & $74.17 \%$ & $71.67 \%$ \\
\hline Mean & & & $78.38 \%$ & $84.62 \%$ & $73.79 \%$ & $75.29 \%$ \\
\hline
\end{tabular}


Table 4. Feature representation-based transfer learning classification results of different pre-trained CNN models using $90 \mathrm{~s}$ measurement duration for the resting state of $\triangle \mathrm{HbO}$

\begin{tabular}{|c|c|c|c|c|c|c|}
\hline \multirow{2}{*}{ CNN model } & \multirow{2}{*}{ Feature layer } & \multirow{2}{*}{ Dataset } & \multicolumn{4}{|c|}{ Averaged Performance(HbO)_90 sec } \\
\hline & & & Accuracy & Recall & Precision & F1-score \\
\hline \multirow{4}{*}{ VGG16 } & \multirow{2}{*}{ fc 6} & $80 \%$ training & $67.78 \%$ & $75.21 \%$ & $60.00 \%$ & $59.21 \%$ \\
\hline & & $70 \%$ training & $74.29 \%$ & $80.60 \%$ & $70.00 \%$ & $71.80 \%$ \\
\hline & \multirow{2}{*}{ fc7 } & $80 \%$ training & $74.89 \%$ & $77.62 \%$ & $72.00 \%$ & $73.11 \%$ \\
\hline & & $70 \%$ training & $72.50 \%$ & $79.99 \%$ & $65.00 \%$ & $67.70 \%$ \\
\hline \multirow{2}{*}{ Resnet50 } & \multirow{2}{*}{ fc1000 } & $80 \%$ training & $67.56 \%$ & $73.17 \%$ & $64.00 \%$ & $62.01 \%$ \\
\hline & & $70 \%$ training & $81.96 \%$ & $83.55 \%$ & $82.50 \%$ & $81.72 \%$ \\
\hline \multirow{2}{*}{ Resnet18 } & \multirow{2}{*}{ fc1000 } & $80 \%$ training & $75.56 \%$ & $77.32 \%$ & $80.00 \%$ & $76.34 \%$ \\
\hline & & $70 \%$ training & $65.00 \%$ & $66.47 \%$ & $60.00 \%$ & $61.46 \%$ \\
\hline \multirow{2}{*}{ Resnet101 } & \multirow{2}{*}{ fc1000 } & $80 \%$ training & $73.56 \%$ & $75.82 \%$ & $76.00 \%$ & $72.35 \%$ \\
\hline & & $70 \%$ training & $63.04 \%$ & $72.34 \%$ & $57.50 \%$ & $56.70 \%$ \\
\hline \multirow{2}{*}{ Densenet201 } & \multirow{2}{*}{ fc1000 } & $80 \%$ training & $90.44 \%$ & $89.92 \%$ & $92.00 \%$ & $90.42 \%$ \\
\hline & & $70 \%$ training & $74.82 \%$ & $76.21 \%$ & $72.50 \%$ & $73.99 \%$ \\
\hline \multirow{4}{*}{ VGG19 } & \multirow{2}{*}{ fc6 } & $80 \%$ training & $78.22 \%$ & $81.10 \%$ & $72.00 \%$ & $75.58 \%$ \\
\hline & & $70 \%$ training & $78.57 \%$ & $78.01 \%$ & $80.00 \%$ & $78.64 \%$ \\
\hline & \multirow{2}{*}{ fc7 } & $80 \%$ training & $76.89 \%$ & $76.14 \%$ & $76.00 \%$ & $75.62 \%$ \\
\hline & & $70 \%$ training & $75.71 \%$ & $79.82 \%$ & $70.00 \%$ & $73.86 \%$ \\
\hline \multirow{4}{*}{ Alexnet } & \multirow{2}{*}{ fc6 } & $80 \%$ training & $74.89 \%$ & $83.17 \%$ & $72.00 \%$ & $70.39 \%$ \\
\hline & & $70 \%$ training & $73.57 \%$ & $87.75 \%$ & $60.00 \%$ & $64.65 \%$ \\
\hline & \multirow{2}{*}{ fc7 } & $80 \%$ training & $77.11 \%$ & $78.01 \%$ & $72.00 \%$ & $72.11 \%$ \\
\hline & & $70 \%$ training & $70.89 \%$ & $85.25 \%$ & $57.50 \%$ & $63.98 \%$ \\
\hline Mean & & & $74.36 \%$ & $78.87 \%$ & $70.55 \%$ & $71.08 \%$ \\
\hline
\end{tabular}

Table 5. Feature representation-based transfer learning classification results of different pre-trained CNN models using $90 \mathrm{~s}$ measurement duration for the resting state of $\Delta \mathrm{HbR}$

\begin{tabular}{|c|c|c|c|c|c|c|}
\hline \multirow{2}{*}{ CNN model } & \multirow{2}{*}{ Feature layer } & \multirow{2}{*}{ Dataset } & \multicolumn{4}{|c|}{ Averaged Performance(HbR)_90 sec } \\
\hline & & & Accuracy & Recall & Precision & F1-score \\
\hline \multirow{4}{*}{ VGG16 } & \multirow{2}{*}{ fc6 } & $80 \%$ training & $79.38 \%$ & $87.21 \%$ & $80.12 \%$ & $85.65 \%$ \\
\hline & & $70 \%$ training & $78.72 \%$ & $80.83 \%$ & $87.50 \%$ & $69.65 \%$ \\
\hline & \multirow{2}{*}{ fc7 } & $80 \%$ training & $64.68 \%$ & $66.58 \%$ & $70.35 \%$ & $56.81 \%$ \\
\hline & & $70 \%$ training & $57.28 \%$ & $67.14 \%$ & $65.06 \%$ & $55.31 \%$ \\
\hline \multirow{2}{*}{ Resnet50 } & \multirow{2}{*}{ fc1000 } & $80 \%$ training & $80.42 \%$ & $82.56 \%$ & $67.00 \%$ & $79.83 \%$ \\
\hline & & $70 \%$ training & $79.51 \%$ & $82.59 \%$ & $70.08 \%$ & $69.83 \%$ \\
\hline \multirow{2}{*}{ Resnet18 } & \multirow{2}{*}{ fc1000 } & $80 \%$ training & $78.87 \%$ & $81.56 \%$ & $51.77 \%$ & $51.83 \%$ \\
\hline & & $70 \%$ training & $81.65 \%$ & $74.29 \%$ & $70.87 \%$ & $67.33 \%$ \\
\hline \multirow{2}{*}{ Resnet101 } & \multirow{2}{*}{ fc1000 } & $80 \%$ training & $79.70 \%$ & $75.29 \%$ & $82.97 \%$ & $60.51 \%$ \\
\hline & & $70 \%$ training & $70.72 \%$ & $78.71 \%$ & $70.45 \%$ & $54.01 \%$ \\
\hline
\end{tabular}




\begin{tabular}{|c|c|c|c|c|c|c|}
\hline \multirow{2}{*}{ Densenet201 } & \multirow{2}{*}{ fc1000 } & $80 \%$ training & $83.25 \%$ & $83.78 \%$ & $84.86 \%$ & $56.51 \%$ \\
\hline & & $70 \%$ training & $72.87 \%$ & $69.42 \%$ & $78.06 \%$ & $51.51 \%$ \\
\hline \multirow{4}{*}{ VGG19 } & \multirow{2}{*}{ fc6 } & $80 \%$ training & $69.86 \%$ & $72.29 \%$ & $53.71 \%$ & $74.71 \%$ \\
\hline & & $70 \%$ training & $74.91 \%$ & $73.56 \%$ & $85.00 \%$ & $66.71 \%$ \\
\hline & \multirow{2}{*}{$\mathrm{fc} 7$} & $80 \%$ training & $65.13 \%$ & $78.12 \%$ & $70.59 \%$ & $56.81 \%$ \\
\hline & & $70 \%$ training & $64.96 \%$ & $76.75 \%$ & $71.69 \%$ & $65.31 \%$ \\
\hline \multirow{4}{*}{ Alexnet } & \multirow{2}{*}{ fc6 } & $80 \%$ training & $68.56 \%$ & $79.51 \%$ & $71.65 \%$ & $69.03 \%$ \\
\hline & & $70 \%$ training & $71.69 \%$ & $74.09 \%$ & $78.30 \%$ & $70.03 \%$ \\
\hline & \multirow{2}{*}{ fc7 } & $80 \%$ training & $77.84 \%$ & $82.57 \%$ & $67.86 \%$ & $75.45 \%$ \\
\hline & & $70 \%$ training & $75.07 \%$ & $77.42 \%$ & $75.78 \%$ & $75.61 \%$ \\
\hline \multicolumn{3}{|l|}{ Mean } & $73.75 \%$ & $77.21 \%$ & $72.68 \%$ & $65.62 \%$ \\
\hline
\end{tabular}

Table 6. Classification-based transfer learning classification results of different pretrained CNN models using $30 \mathrm{~s}$ measurement duration for the resting state of $\Delta \mathrm{HbO}$

\begin{tabular}{lllllll}
\hline \multirow{2}{*}{ CNN model } & \multirow{2}{*}{ Altered layer } & \multirow{2}{*}{ Dataset } & \multicolumn{4}{c}{ Averaged Performance(HbO)_30 sec } \\
\cline { 4 - 6 } & & & Accuracy & Recall & Precision & F1-score \\
\hline \multirow{2}{*}{ Alexnet } & \multirow{2}{*}{ Layer23/25 } & $80 \%$ training & $87.45 \%$ & $88.75 \%$ & $87.50 \%$ & $86.54 \%$ \\
& \multirow{2}{*}{ VGG16 } & $70 \%$ training & $84.58 \%$ & $87.61 \%$ & $81.67 \%$ & $83.53 \%$ \\
& \multirow{2}{*}{ Layer39/41 } & $80 \%$ training & $92.43 \%$ & $91.47 \%$ & $93.75 \%$ & $92.46 \%$ \\
& \multirow{2}{*}{ VGG19 } & $70 \%$ training & $88.58 \%$ & $94.89 \%$ & $81.67 \%$ & $86.76 \%$ \\
& \multirow{2}{*}{ Layer45/47 } & $80 \%$ training & $94.40 \%$ & $96.40 \%$ & $92.50 \%$ & $94.28 \%$ \\
& & $70 \%$ training & $85.00 \%$ & $89.12 \%$ & $80.00 \%$ & $83.99 \%$ \\
\hline
\end{tabular}

Table 7. Classification-based transfer learning classification results of different pretrained CNN models using $30 \mathrm{~s}$ measurement duration for the resting state of $\Delta \mathrm{HbR}$

\begin{tabular}{|c|c|c|c|c|c|c|}
\hline \multirow{2}{*}{ CNN model } & \multirow{2}{*}{ Altered layer } & \multirow{2}{*}{ Dataset } & \multicolumn{4}{|c|}{ Averaged Performance(HbR)_30 sec } \\
\hline & & & Accuracy & Recall & Precision & F1-score \\
\hline \multirow{2}{*}{ Alexnet } & \multirow{2}{*}{ Layer23/25 } & $80 \%$ training & $95.30 \%$ & $92.56 \%$ & $98.75 \%$ & $95.51 \%$ \\
\hline & & $70 \%$ training & $91.33 \%$ & $91.28 \%$ & $91.67 \%$ & $91.19 \%$ \\
\hline \multirow{2}{*}{ VGG16 } & \multirow{2}{*}{ Layer39/41 } & $80 \%$ training & $95.14 \%$ & $97.58 \%$ & $92.50 \%$ & $94.77 \%$ \\
\hline & & $70 \%$ training & $88.08 \%$ & $96.77 \%$ & $79.17 \%$ & $86.51 \%$ \\
\hline \multirow{2}{*}{ VGG19 } & \multirow{2}{*}{ Layer45/47 } & $80 \%$ training & $97.01 \%$ & $97.76 \%$ & $96.25 \%$ & $91.14 \%$ \\
\hline & & $70 \%$ training & $92.17 \%$ & $98.36 \%$ & $85.83 \%$ & $91.14 \%$ \\
\hline Mean & & & $93.17 \%$ & $95.72 \%$ & $90.69 \%$ & $91.71 \%$ \\
\hline
\end{tabular}

Table 8. Classification-based transfer learning classification results of different pretrained CNN models using $90 \mathrm{~s}$ measurement duration for the resting state of $\Delta \mathrm{HbO}$

\begin{tabular}{llllll}
\hline \multirow{2}{*}{ CNN model } & Altered layer & Dataset & \multicolumn{4}{l}{ Averaged Performance(HbO)_90 sec } \\
\cline { 3 - 6 } & & Accuracy & Recall & Precision & F1-score \\
\hline
\end{tabular}




\begin{tabular}{lclllll}
\hline \multirow{2}{*}{ Alexnet } & \multirow{2}{*}{ Layer23/25 } & $80 \%$ training & $73.78 \%$ & $93.75 \%$ & $52.00 \%$ & $80.93 \%$ \\
& & $70 \%$ training & $78.39 \%$ & $72.62 \%$ & $92.50 \%$ & $80.93 \%$ \\
\multirow{2}{*}{ VGG16 } & \multirow{2}{*}{ Layer39/41 } & $80 \%$ training & $70.44 \%$ & $77.87 \%$ & $52.00 \%$ & $81.70 \%$ \\
& & $70 \%$ training & $80.18 \%$ & $77.86 \%$ & $87.50 \%$ & $81.70 \%$ \\
\multirow{2}{*}{ VGG19 } & \multirow{2}{*}{ Layer45/47 } & $80 \%$ training & $67.33 \%$ & $65.68 \%$ & $68.00 \%$ & $68.54 \%$ \\
& & $70 \%$ training & $72.32 \%$ & $74.05 \%$ & $67.50 \%$ & $68.54 \%$ \\
\hline Mean & & & $73.74 \%$ & $76.97 \%$ & $69.92 \%$ & $77.06 \%$ \\
\hline
\end{tabular}

Table 9. Classification-based transfer learning classification results of different pretrained CNN models using $90 \mathrm{~s}$ measurement duration for the resting state of $\Delta \mathrm{HbR}$

\begin{tabular}{lllllll}
\hline \multirow{2}{*}{ CNN model } & \multirow{2}{*}{ Altered layer } & \multirow{2}{*}{ Dataset } & \multicolumn{4}{c}{ Averaged Performance(HbR)_90 sec } \\
\cline { 4 - 6 } & & & Accuracy & Recall & Precision & F1-score \\
\hline \multirow{2}{*}{ Alexnet } & \multirow{2}{*}{ Layer23/25 } & $80 \%$ training & $75.36 \%$ & $75.09 \%$ & $80.00 \%$ & $76.21 \%$ \\
& & $70 \%$ training & $81.11 \%$ & $81.06 \%$ & $79.48 \%$ & $71.71 \%$ \\
\multirow{2}{*}{ VGG16 } & \multirow{2}{*}{ Layer39/41 } & $80 \%$ training & $78.69 \%$ & $83.86 \%$ & $78.00 \%$ & $83.83 \%$ \\
& \multirow{2}{*}{ VGG19 } & $70 \%$ training & $81.47 \%$ & $82.26 \%$ & $73.73 \%$ & $76.33 \%$ \\
& \multirow{2}{*}{ Layer45/47 } & $80 \%$ training & $72.03 \%$ & $85.29 \%$ & $53.85 \%$ & $74.35 \%$ \\
\hline \multirow{2}{*}{ Mean } & $70 \%$ training & $75.22 \%$ & $77.94 \%$ & $60.23 \%$ & $64.35 \%$ \\
\hline
\end{tabular}

\section{List of Figures}

Figure 1. System flowchart of the entire experimental process for the current study.

Figure 2. (a) Arrangement of the emitters and detectors; (b) channel configuration.

Figure 3. Schematic of the transfer learning algorithm: (a) feature representation-based transfer learning, (b) classification-based transfer learning.

Figure 4. Classification results of the linear discrimination analysis, support vector machine, and K-nearest neighbor algorithms for nine measurement durations $(0-30,0-$ 60, 0-90, 0-120, 0-150, 0-180, 0-210, 0-240, and 0-270 s).

Figure 5. Connectivity maps of the resting state for the MCI and $\mathrm{HC}$ groups during nine measurement durations (i.e., 0-30, 0-60, 0-90, 0-120, 0-150,0-180, 0-210, 0-240, and 0-270 s).

Figure 6. Graph theory parameters of the resting-state functional connectivity of the MCI and $\mathrm{HC}$ groups for nine measurement durations (i.e., 0-30, 0-60, 0-90, 0-120, 0-150, 0 180, 0-210, 0-240, and 0-270 s): (a) global efficiency, (b) local efficiency, and (c) smallworldness. 


\section{References}

1. Mattson MP. Pathways towards and away from Alzheimer's disease. Nature. 2004;430:631-639.

2. Alzheimer's Association. 2020 Alzheimer's disease facts and figures. Alzheimer's Dement. 2020;16:391-460.

3. Wyss-Coray T. Ageing, neurodegeneration and brain rejuvenation. Nature. 2016;539:180-186.

4. Cummings J, Passmore P, McGuinness B, Mok V, Chen C, Engelborghs S, et al. Souvenaid in the management of mild cognitive impairment: An expert consensus opinion. Alzheimer's Research \& Therapy; 2019;11:AN:73.

5. Livingston G, Huntley J, Sommerlad A, Ames D, Ballard C, Banerjee S, et al. Dementia prevention, intervention, and care: 2020 report of the Lancet Commission. lancet Comm. Elsevier; 2020;396:413-446.

6. Knopman DS, Petersen RC. Mild cognitive impairment and mild dementia: A clinical perspective. Mayo Clin Proc. Elsevier Inc; 2014;89:1452-1459.

7. Young PNE, Estarellas M, Coomans E, Srikrishna M, Beaumont H, Maass A, et al. Imaging biomarkers in neurodegeneration: Current and future practices. Alzheimer's Research \& Therapy; 2020;12:1-17.

8. Horwitz B, Friston KJ, Taylor JG. Neural modeling and functional brain imaging: An overview. Neural Networks. 2000;13:829-46.

9. Irani F, Platek SM, Bunce S, Ruocco AC, Chute D. Functional near infrared spectroscopy (fNIRS): An emerging neuroimaging technology with important applications for the study of brain disorders. Clin Neuropsychol. 2007;21:9-37.

10. Miladinović D, Muheim C, Bauer S, Spinnler A, Noain D, Bandarabadi M, et al. SPINDLE: End-to-end learning from EEG/EMG to extrapolate animal sleep scoring across experimental settings, labs and species. PLoS Comput Biol. 2019;15:1-30.

11. Freeman WJ, Holmes MD, Burke BC, Vanhatalo S. Spatial spectra of scalp EEG and EMG from awake humans. Clin Neurophysiol. 2003;114:1053-68.

12. Logothetis NK. What we can do and what we cannot do with fMRI. Nature. 2008;453:869-78.

13. Scarapicchia V, Brown C, Mayo C, Gawryluk JR. Functional magnetic resonance imaging and functional near-infrared spectroscopy: Insights from combined recording studies. Front Hum Neurosci. 2017;11:1-12.

14. Hong K.-S., Yaqub MA. Application of functional near-infrared spectroscopy in the healthcare industry: A review. J Innov Opt Heal Sci. 2019; 12:06, AN: 1030012.

15. Naseer N, Hong K.-S. fNIRS-based brain-computer interfaces: a review. Front Hum Neurosci. 2015;9; AN:003.

16. Hong K.-S., Ghafoor U, Khan MJ. Brain-machine interfaces using functional nearinfrared spectroscopy: a review. Artif Life Robot. Springer Japan; 2020;25: 204-218.

17. Hong K.-S., Zafar A. Existence of Initial Dip for BCI: An Illusion or Reality. Front Neurorobot. 2018;12; AN:69.

18. Khan MNA, Bhutta MR, Hong K.-S.. Task-Specific Stimulation Duration for fNIRS Brain-Computer Interface. IEEE Access. 2020;8:89093-89105.

19. Hong K.-S., Naseer N. Reduction of Delay in Detecting Initial Dips from Functional Near-Infrared Spectroscopy Signals Using Vector-Based Phase Analysis. Int J Neural Syst. 2016;26; AN:1650012.

20. Nguyen H.-D., Hong K.-S., Shin Y-I. Bundled-Optode Method in Functional Near- 
Infrared Spectroscopy. PLoS One. 2016;11; AN: e0165146.

21. Hong K.-S., Khan MJ, Hong MJ. Feature Extraction and Classification Methods for Hybrid fNIRS-EEG Brain-Computer Interfaces. Front Hum Neurosci. 2018;12; AN:246.

22. Nguyen H.-D. and Hong K.-S. Bundled-optode implementation for 3D imaging in functional near-infrared spectroscopy. Biomed Opt Express. 2016;7:3491-507.

23. Hong K.-S. and Nguyen H.-D. State-space models of impulse hemodynamic responses over motor, somatosensory, and visual cortices. Biomed Opt Express. 2014;5; AN:1778.

24. Turnip A, Hong K.-S., Jeong M.-Y. Real-time feature extraction of P300 component using adaptive nonlinear principal component analysis. Biomed Eng Online. 2011;10; AN: 83.

25. Nguyen H.-D., Yoo S.-H., Bhutta MR, Hong K.-S. Adaptive filtering of physiological noises in fNIRS data. Biomed Eng Online. 2018;17:4-9.

26. Hong K.-S., Pham PT. Control of Axially Moving Systems: A Review. Int J Control Autom Syst. 2019;17:2983-3008.

27. Pamosoaji AK, Piao M, Hong K.-S.. PSO-based Minimum-time Motion Planning for Multiple Vehicles Under Acceleration and Velocity Limitations. Int J Control Autom Syst. 2019;17:2610-2623.

28. Yeung MK, Chan AS. Functional near-infrared spectroscopy reveals decreased resting oxygenation levels and task-related oxygenation changes in mild cognitive impairment and dementia: A systematic review. J Psychiatr Res. Elsevier Ltd; 2020;124:58-76.

29. Bu L, Huo C, Qin Y, Xu G, Wang Y, Li Z. Effective Connectivity in Subjects with Mild Cognitive Impairment as Assessed Using Functional Near-Infrared Spectroscopy. Am J Phys Med Rehabil. 2019;98:438-45.

30. Nguyen T, Kim M, Gwak J, Lee JJ, Choi KY, Lee KH, et al. Investigation of brain functional connectivity in patients with mild cognitive impairment: A functional near-infrared spectroscopy (fNIRS) study. J Biophotonics. 2019;12:1-10.

31. Niu $\mathrm{H}$, Zhu Z. Abnormal dynamic functional connectivity and brain states in Alzheimer's diseases: functional near-infrared spectroscopy study. Neurophotonics. 2019;6:1.

32. Viola S, Viola P, Buongarzone MP, Fiorelli L, Litterio P. Tissue oxygen saturation and pulsatility index as markers for amnestic mild cognitive impairment: NIRS and TCD study. Clin Neurophysiol. International Federation of Clinical Neurophysiology; 2013;124:851-6.

33. Liu J, Zhu YS, Khan MA, Brunk E, Martin-Cook K, Weiner MF, et al. Global brain hypoperfusion and oxygenation in amnestic mild cognitive impairment. Alzheimer's Dement. 2014;10:162-170.

34. Babiloni C, Vecchio F, Altavilla R, Tibuzzi F, Lizio R, Altamura C, et al. Hypercapnia affects the functional coupling of resting state electroencephalographic rhythms and cerebral haemodynamics in healthy elderly subjects and in patients with amnestic mild cognitive impairment. Clin Neurophysiol. International Federation of Clinical Neurophysiology; 2014;125:685-693.

35. Marmarelis VZ, Shin DC, Tarumi T, Zhang R. Comparison of model-based indices of cerebral autoregulation and vasomotor reactivity using transcranial doppler versus near-infrared spectroscopy in patients with amnestic mild cognitive impairment. $J$ Alzheimer's Dis. 2017;56:89-105. 
36. Zeller JBM, Katzorke A, Müller LD, Breunig J, Haeussinger FB, Deckert J, et al. Reduced spontaneous low frequency oscillations as measured with functional nearinfrared spectroscopy in mild cognitive impairment. Brain Imaging Behav. 2019;13:283-92.

37. Yap KH, Ung WC, Ebenezer EGM, Nordin N, Chin PS, Sugathan S, et al. Visualizing hyperactivation in neurodegeneration based on prefrontal oxygenation: A comparative study of mild Alzheimer's disease, mild cognitive impairment, and healthy controls. Front Aging Neurosci. 2017;9; AN:287.

38. Katzorke A, Zeller JBM, Müller LD, Lauer M, Polak T, Deckert J, et al. Decreased hemodynamic response in inferior frontotemporal regions in elderly with mild cognitive impairment. Psychiatry Res - Neuroimaging; 2018;274:11-18.

39. Plichta MM, Herrmann MJ, Baehne CG, Ehlis AC, Richter MM, Pauli P, et al. Eventrelated functional near-infrared spectroscopy (fNIRS): Are the measurements reliable? Neuroimage. 2006;31:116-124.

40. Yeung MK, Sze SL, Woo J, Kwok T, Shum DHK, Yu R, et al. Reduced Frontal Activations at High Working Memory Load in Mild Cognitive Impairment: NearInfrared Spectroscopy. Dement Geriatr Cogn Disord. 2016;42:278-296.

41. Yeung MK, Sze SL, Woo J, Kwok T, Shum DHK, Yu R, et al. Altered frontal lateralization underlies the category fluency deficits in older adults with mild cognitive impairment: A near-infrared spectroscopy study. Front Aging Neurosci. 2016;8; AN:59.

42. Vermeij A, Kessels RPC, Heskamp L, Simons EMF, Dautzenberg PLJ, Claassen JAHR. Prefrontal activation may predict working-memory training gain in normal aging and mild cognitive impairment. Brain Imaging Behav. 2017;11:141-154.

43. Niu HJ, Li X, Chen YJ, Ma C, Zhang JY, Zhang ZJ. Reduced Frontal Activation during a Working Memory Task in Mild Cognitive Impairment: A Non-Invasive Near-Infrared Spectroscopy Study. CNS Neurosci Ther. 2013;19:125-131.

44. Li R, Rui G, Chen W, Li S, Schulz PE, Zhang Y. Early Detection of Alzheimer's Disease Using Non-invasive Near-Infrared Spectroscopy. Front Aging Neurosci. 2018;10; AN:366.

45. Tak S, Yoon SJ, Jang J, Yoo K, Jeong Y, Ye JC. Quantitative analysis of hemodynamic and metabolic changes in subcortical vascular dementia using simultaneous near-infrared spectroscopy and fMRI measurements. Neuroimage. 2011;55:176-184.

46. Doi T, Makizako H, Shimada H, Park H, Tsutsumimoto K, Uemura K, et al. Brain activation during dual-task walking and executive function among older adults with mild cognitive impairment: A fNIRS study. Aging Clin Exp Res. 2013;25:539-544.

47. Zeller JBM, Herrmann MJ, Ehlis AC, Polak T, Fallgatter AJ. Altered parietal brain oxygenation in alzheimer's disease as assessed with near-infrared spectroscopy. Am J Geriatr Psychiatry. American Association for Geriatric Psychiatry; 2010;18:433441.

48. Kito H, Ryokawa A, Kinoshita Y, Sasayama D, Sugiyama N, Ogihara T, et al. Comparison of alterations in cerebral hemoglobin oxygenation in late life depression and Alzheimer's disease as assessed by near-infrared spectroscopy. Behav Brain Funct. 2014;10; AN:8.

49. Yang D, Huang R, Yoo S.-H., Shin M.-J., Yoon J.-A., Shin Y Il, et al. Detection of Mild Cognitive Impairment Using Convolutional Neural Network: TemporalFeature Maps of Functional Near-Infrared Spectroscopy. Front Aging Neurosci. 
2020;12; AN:141.

50. Yang D, Hong K.-S., Yoo S.-H., Kim C.-S. Evaluation of neural degeneration biomarkers in the prefrontal cortex for early identification of patients with mild cognitive impairment: an fNIRS study. Front Hum Neurosci. 2019;13:317.

51. Han C, Jo S, Jo I, Kim E, Park M, et al. An adaptation of the Korean mini-mental state examination (K-MMSE) in elderly Koreans: demographic influence and population-based norms (the AGE study). Elsevier. 2008;47:302-310.

52. Ahn HJ, Chin J, Park A, Lee BH, Suh MK, Seo SW, et al. Seoul neuropsychological screening battery-dementia version (SNSB-D): A useful tool for assessing and monitoring cognitive impairments in dementia patients. J Korean Med Sci. 2010;25:1071-1076.

53. Kocsis L, Herman P, and Eke A.The modified Beer-Lambert law revisited. Physics in Medicine and Biology.2006; 51:5:N91-98.

54. Sassaroli A, Fantini S. Comment on the modified Beer-Lambert law for scattering media. Phys Med Biol. 2004.49:N255-N257.

55. Naseer N, Noori FM, Qureshi NK, Hong K.-S. Determining optimal featurecombination for LDA classification of functional near-infrared spectroscopy signals in brain-computer interface application. Front Hum Neurosci. 2016;10; AN:237.

56. Khan MJ and Hong K.-S. Hybrid EEG-FNIRS-based eight-command decoding for BCI: Application to quadcopter control. Front Neurorobot. 2017;11; AN:6.

57. Liu X, Kim CS, and Hong K.-S. An fNIRS-based investigation of visual merchandising displays for fashion stores. PLoS One. 2018;13; AN: e0208843.

58. Zhang H, Duan L, Zhang YJ, Lu CM, Liu H, Zhu CZ. Test-retest assessment of independent component analysis-derived resting-state functional connectivity based on functional near-infrared spectroscopy. Neuroimage. 2011;55:607-715.

59. Korhonen V, Hiltunen T, Myllylä T, Wang X, Kantola J, Nikkinen J, et al. Synchronous multiscale neuroimaging environment for critically sampled physiological analysis of brain function: hepta-scan concept. Brain Connect. 2014;4:677-689.

60. Ghafoor U, Lee JH, Hong K.-S., Park SS, Kim J, Yoo HR. Effects of acupuncture therapy on MCI patients using functional near-infrared spectroscopy. Front Aging Neurosci. 2019;11; AN:237.

61. Niu $\mathrm{H}$ and He Y. Resting-state functional brain connectivity: Lessons from functional near-infrared spectroscopy. Neuroscientist. 2014;20:173-188.

62. Wada A, Tsuruta K, Irie R, Kamagata K, Maekawa T, Fujita S, et al. Differentiating Alzheimer's disease from dementia with lewy bodies using a deep learning technique based on structural brain connectivity. Magn Reson Med Sci. 2019;18:219-224.

63. Manohar N. and Shanta M. Machine Learning Techniques to Identify Dementia. SN Comput Sci. 2020;1:AN: 118.

64. Muazzam Maqsood, Faria Nazir, Umair Khan FA. Transfer Learning Assisted Classification and Detection of Alzheimer' s Disease Stages Using 3D. Sensors. 2019;19: 11; AN:2645.

65. Pan SJ, Fellow QY. A Survey on Transfer Learning. IEEE Transactions on Knowledge and Data Engineering. 2010;22:10: 134-1359.

66. Weiss K, Khoshgoftaar TM, Wang D. A survey of transfer learning. J. Big Data. 2016; 6; AN:9.

67. Vemuri P, Jones DT, Jr RJ. Resting state functional MRI in Alzheimer's Disease. Alzheimer's Research \& Therapy.2012;4:AN:2. 
68. Briels CT, Schoonhoven DN, Stam CJ, Waal H De, Scheltens P, Gouw AA. Reproducibility of EEG functional connectivity in Alzheimer's disease. Alzheimer's Research \& Therapy; 2020;3;AN:68.

69. Tak S, Ye JC. Statistical analysis of fNIRS data: A comprehensive review. Neuroimage. 2014;85:72-91.

70. Vieira S, Pinaya WHL, Mechelli A. Neuroscience and Biobehavioral Reviews Using deep learning to investigate the neuroimaging correlates of psychiatric and neurological disorders: Methods and applications. Neurosci Biobehav Rev. 2017;74:58-75.

71. Markus W, Umut D, Sebastian K, Gerald S, Daniela S, Johannes K, et al. Nuclear Medicine Diagnostic Techniques in the Era of Pathophysiology-Based CSF Biomarkers for Alzheimer' s Disease. J Alzheimers Dis. 2011;26:97-103.

72. Wang J, Dong Q, Niu H. The minimum resting-state fNIRS imaging duration for accurate and stable mapping of brain connectivity network in children. Sci Rep. 2017; 7; AN: 6461. 
Figures

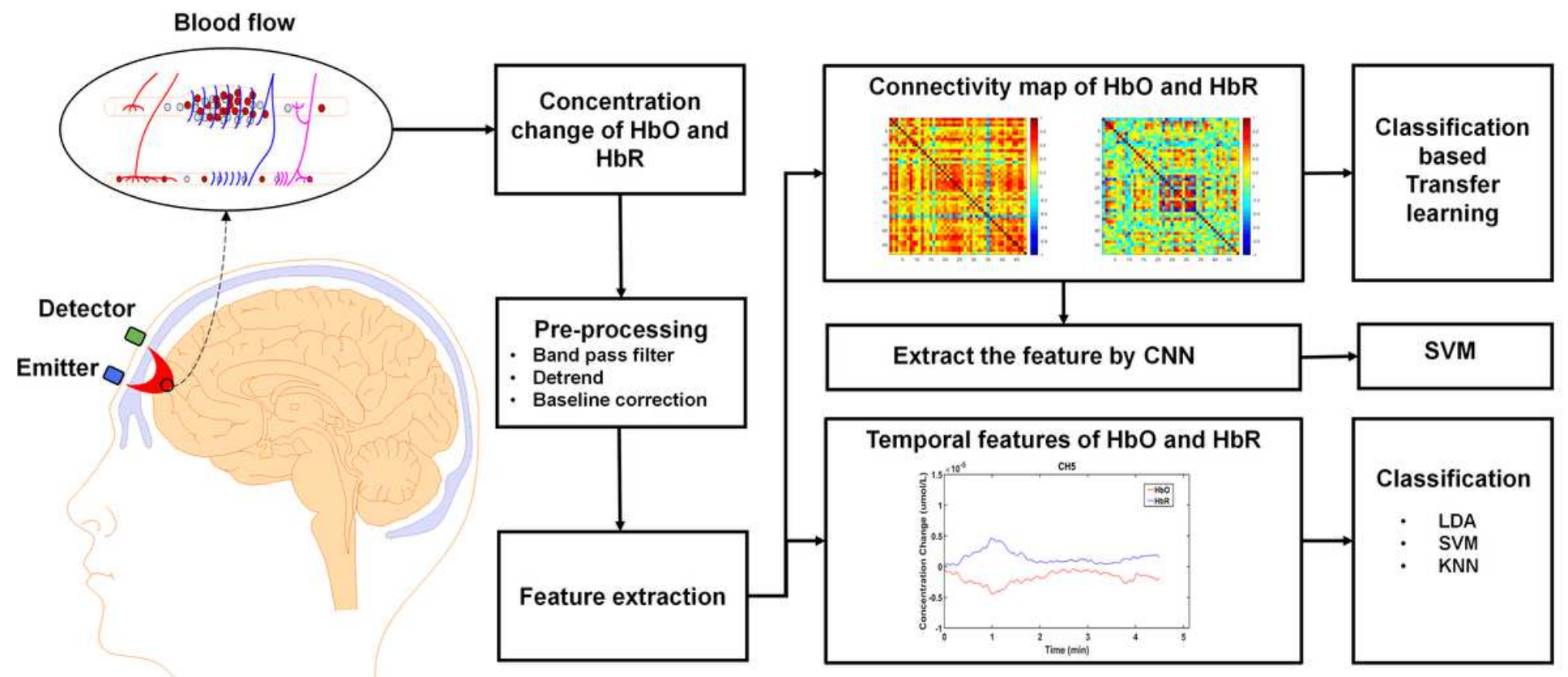

Figure 1

System flowchart of the entire experimental process for the current study.

(a)

(b)
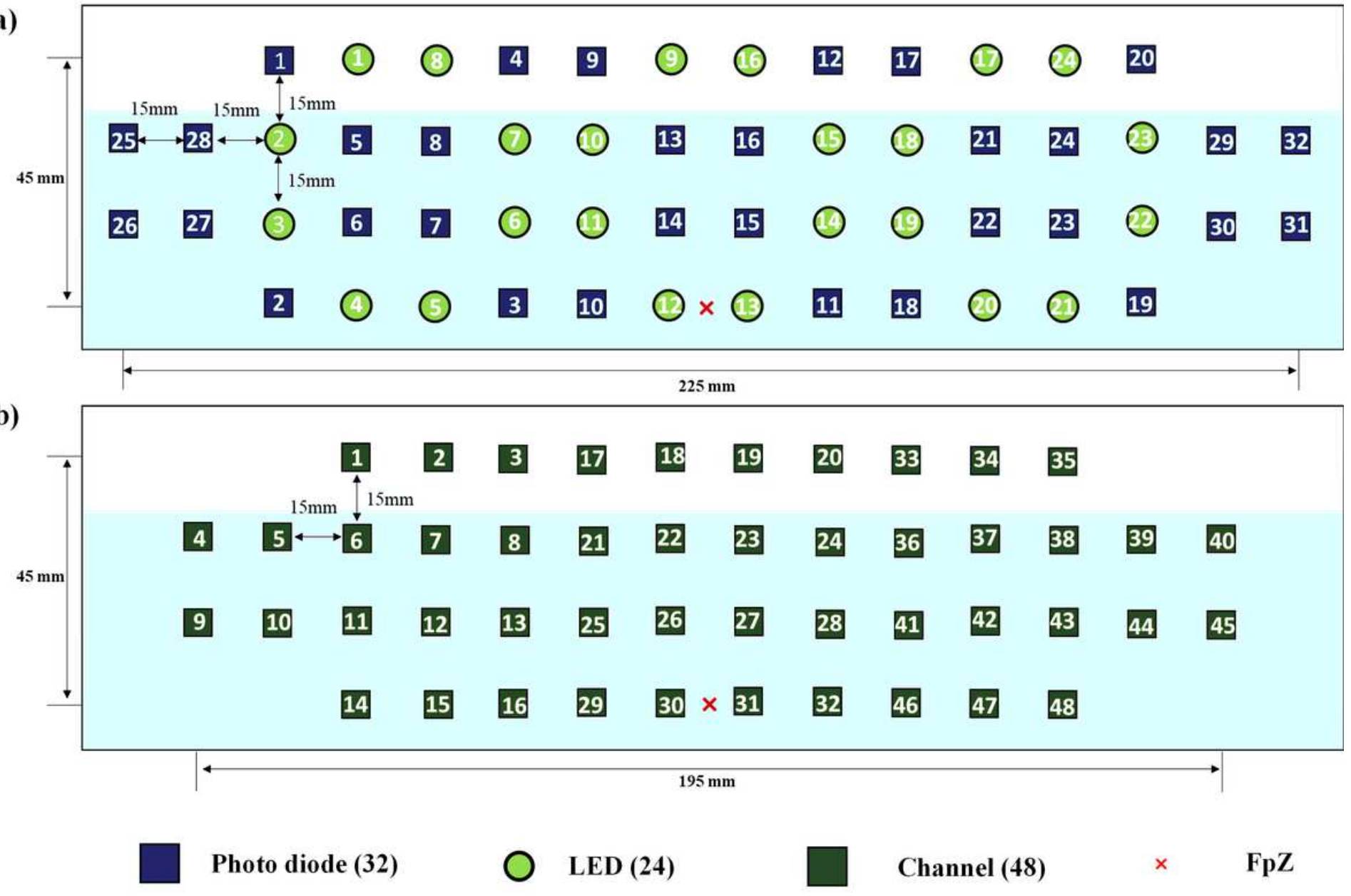
Figure 2

(a) Arrangement of the emitters and detectors; (b) channel configuration.

(a)
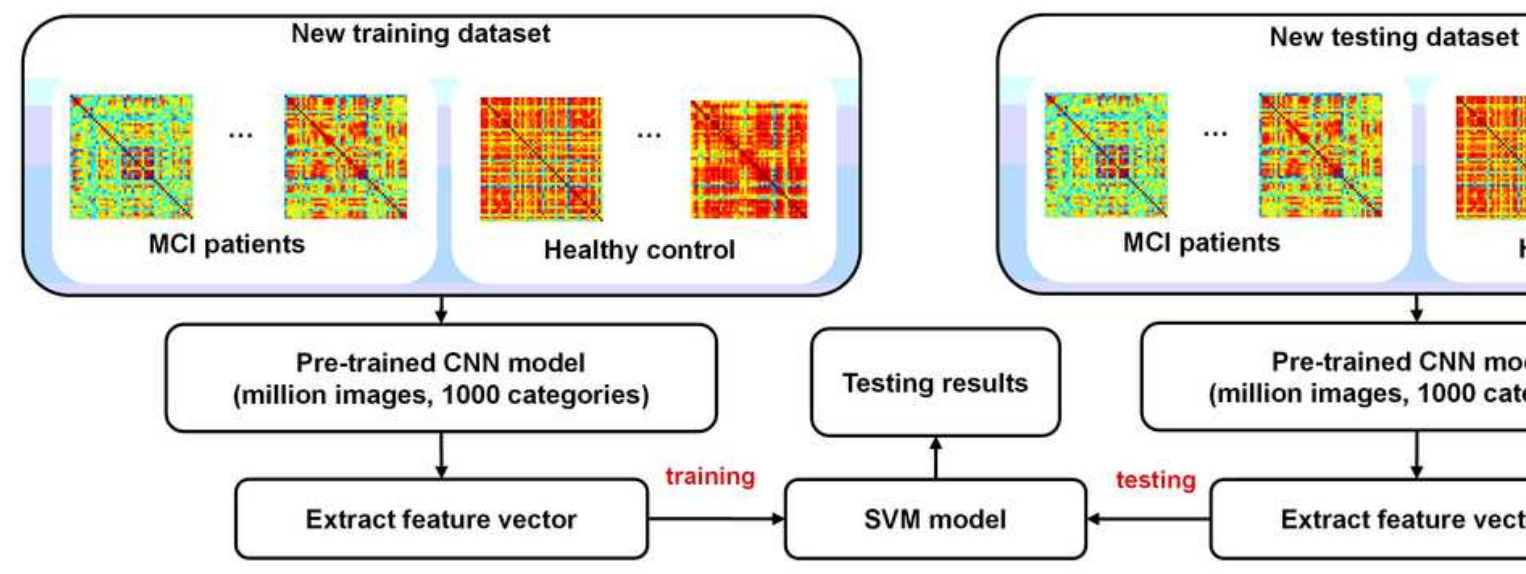

$\mathrm{MCl}$ patients

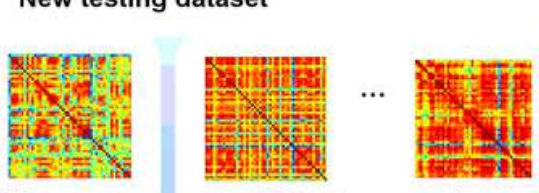

Healthy control

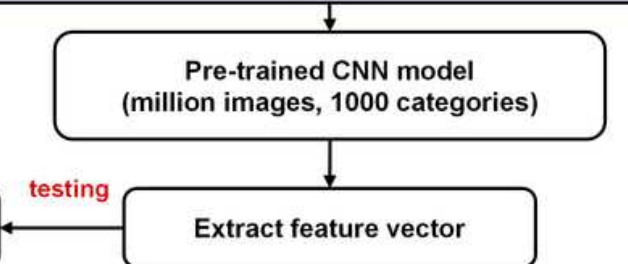

(b)

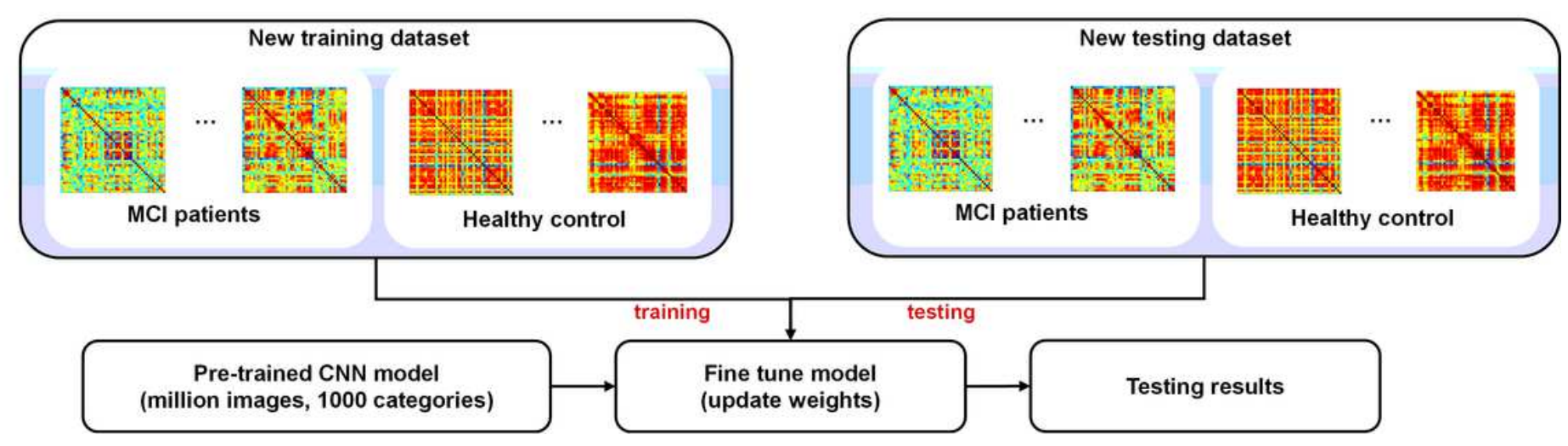

\section{Figure 3}

Schematic of the transfer learning algorithm: (a) feature representation-based transfer learning, (b) classification-based transfer learning. 


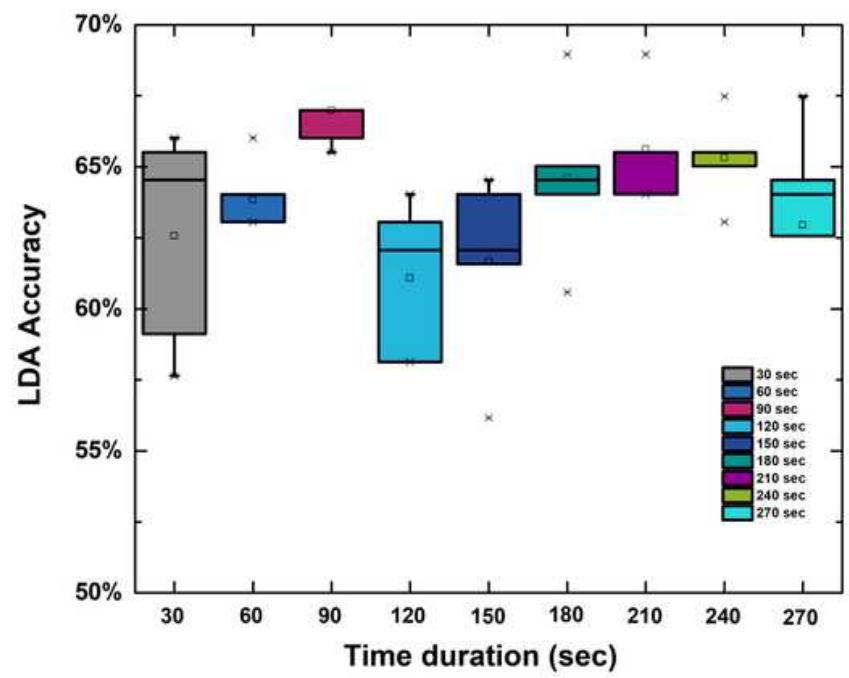

(a)

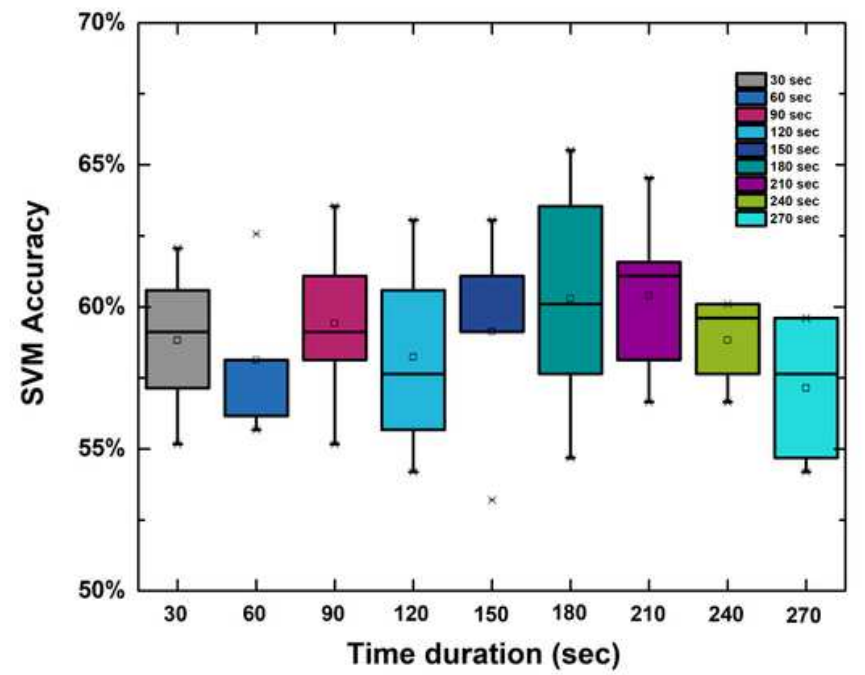

(b)

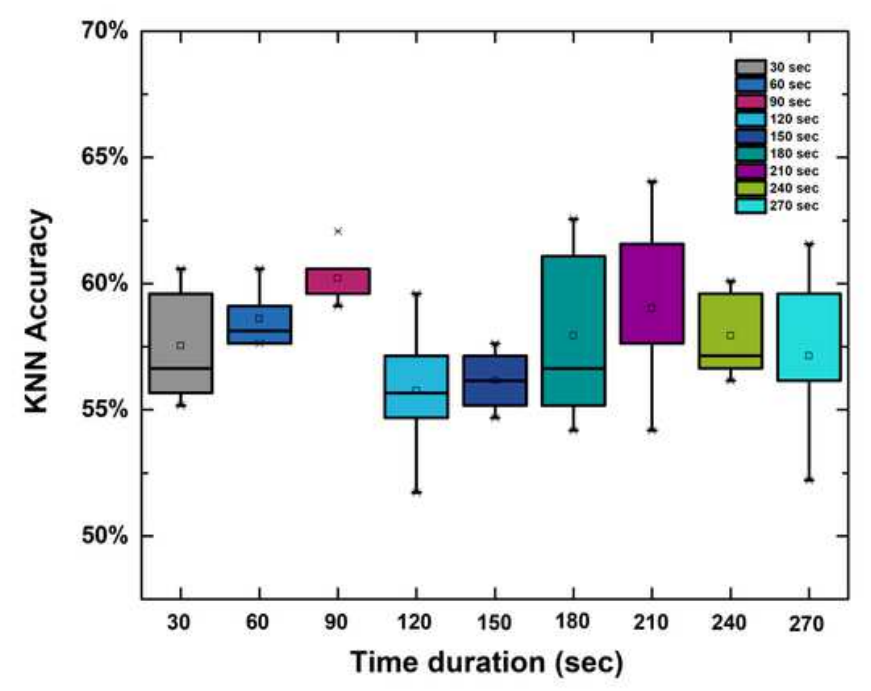

(c)

\section{Figure 4}

Classification results of the linear discrimination analysis, support vector machine, and K-nearest neighbor algorithms for nine measurement durations $(0-30,0-60,0-90,0-120,0-150,0-180,0-210$, 0-240, and 0-270 s). 


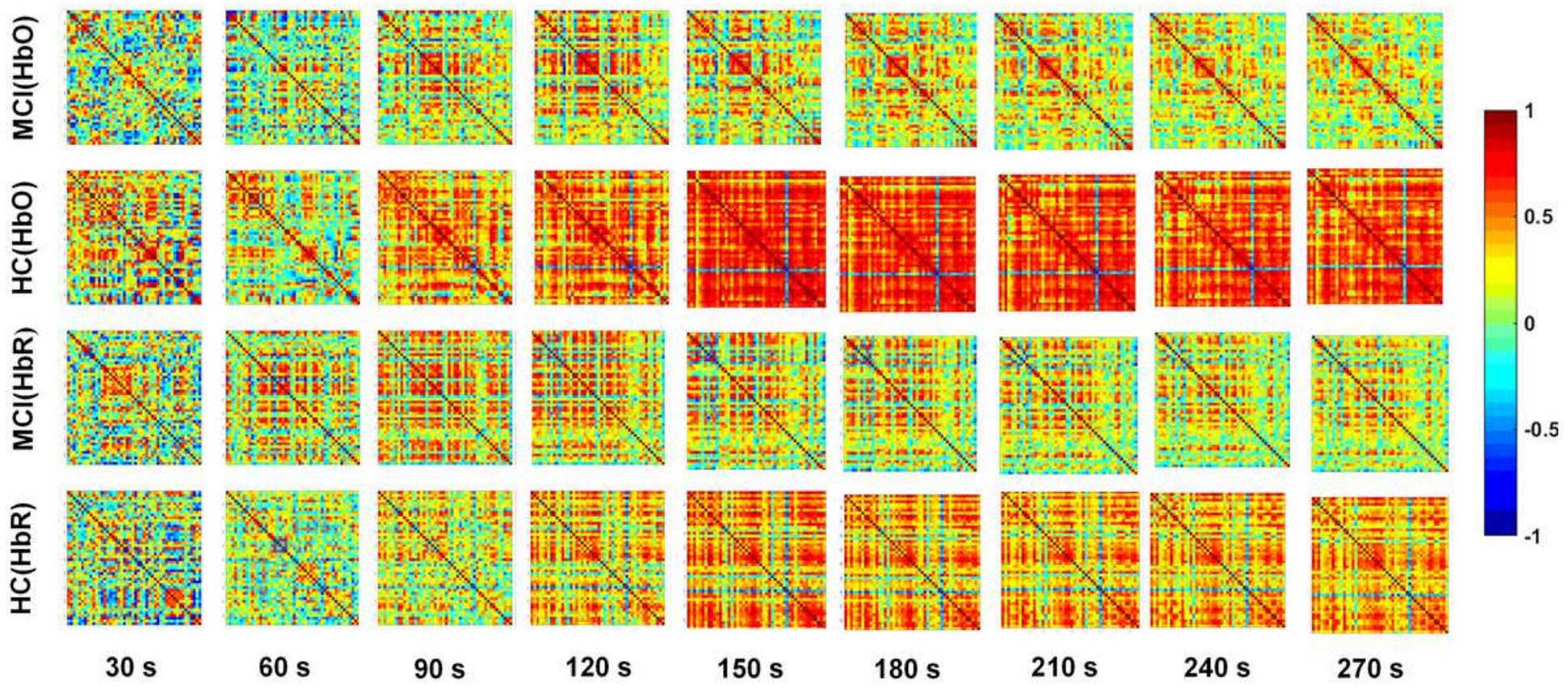

Figure 5

Connectivity maps of the resting state for the $\mathrm{MCl}$ and $\mathrm{HC}$ groups during nine measurement durations (i.e., $0-30,0-60,0-90,0-120,0-150,0-180,0-210,0-240$, and 0-270 s). 


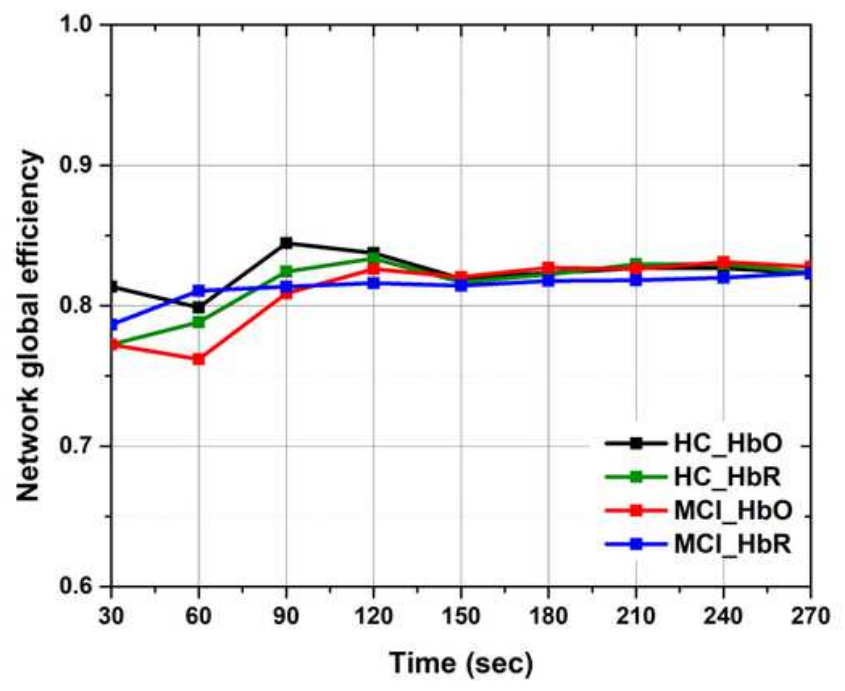

(a)

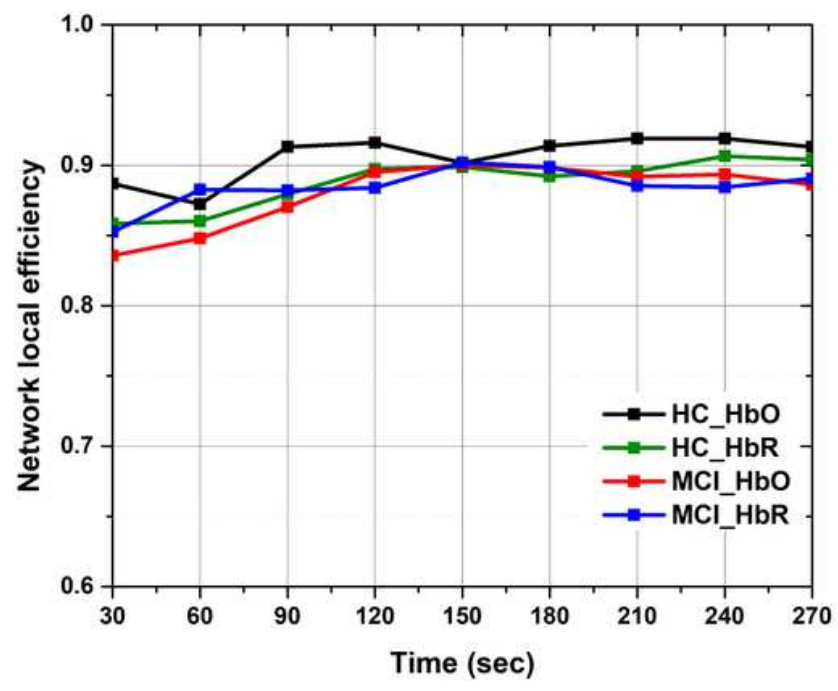

(b)

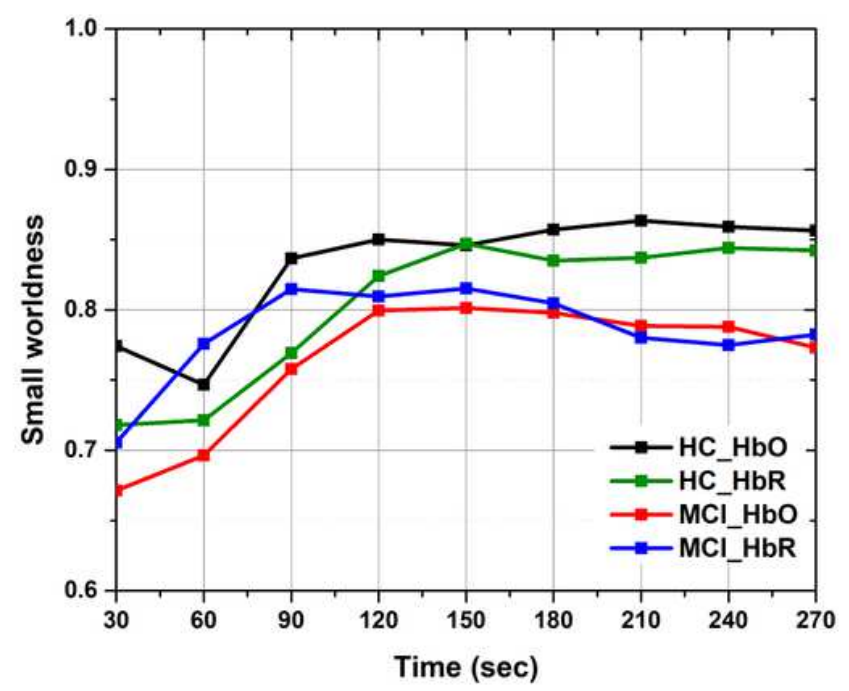

(c)

\section{Figure 6}

Graph theory parameters of the resting-state functional connectivity of the $\mathrm{MCl}$ and $\mathrm{HC}$ groups for nine measurement durations (i.e., $0-30,0-60,0-90,0-120,0-150,0-180,0-210,0-240$, and 0-270 s): (a) global efficiency, (b) local efficiency, and (c) small-worldness. 